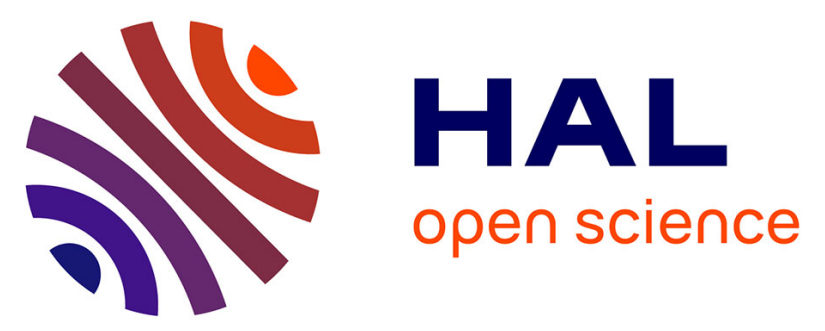

\title{
Two-Photon Absorption in a Conformationally Twisted D- $\pi$-A Oligomer: A Synergic Photosensitizing Approach for Multiphoton Lithography
}

Jean-Pierre Malval, Sylvain Achelle, Loïc Bodiou, Arnaud Spangenberg, Laura Chia Gomez, Olivier Soppera, Françoise Robin-Le Guen

\section{- To cite this version:}

Jean-Pierre Malval, Sylvain Achelle, Loïc Bodiou, Arnaud Spangenberg, Laura Chia Gomez, et al.. Two-Photon Absorption in a Conformationally Twisted D- $\pi$-A Oligomer : A Synergic Photosensitizing Approach for Multiphoton Lithography. Journal of Materials Chemistry C, 2014, 2 (37), pp.7869-7880. 10.1039/C4TC00841C . hal-01089207

\section{HAL Id: hal-01089207 https://hal.science/hal-01089207}

Submitted on 3 Dec 2014

HAL is a multi-disciplinary open access archive for the deposit and dissemination of scientific research documents, whether they are published or not. The documents may come from teaching and research institutions in France or abroad, or from public or private research centers.
L'archive ouverte pluridisciplinaire HAL, est destinée au dépôt et à la diffusion de documents scientifiques de niveau recherche, publiés ou non, émanant des établissements d'enseignement et de recherche français ou étrangers, des laboratoires publics ou privés. 


\section{Two-Photon Absorption in a Conformationally Twisted D- $\pi$-A Oligomer : A Synergic Photosensitizing Approach for Multiphoton Lithography}

Jean-Pierre Malval, ${ }^{a}$ Sylvain Achelle, ${ }^{* b}$ Loic Bodiou, ${ }^{c}$ Arnaud Spangenberg, ${ }^{a}$ Laura Chia Gomez, ${ }^{a}$ Olivier Soppera ${ }^{a}$ and Françoise Robin-le Guen. ${ }^{b}$

${ }^{a}$ Institut de Science des Matériaux de Mulhouse, UMR CNRS 7361, Université de Haute-Alsace, 15 rue Jean Starcky, 68057 Mulhouse, France E-Mail : jean-pierre.malval@uha.fr; Tel : +33 (0)389608 769

${ }^{b}$ Institut des Sciences Chimiques de Rennes UMR CNRS 6226, IUT de Lannion, rue Edouard Branly, BP 30219, F22302 Lannion Cedex, France E-Mail : sylvain.achelle@univ-rennes1.fr; Tel : +33 (0)296469448

${ }^{c}$ Université Européenne de Bretagne (UEB) CNRS, FOTON, UMR 6082, CCLO, ENSSAT BP80518, 22305 Lannion cedex, France

\section{TABLE OF CONTENT ENTRY}

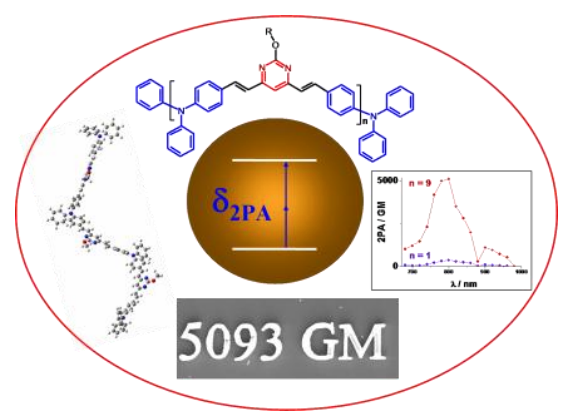

The synthesis and the photophysical properties of a $\pi$-conjugated oligomer alternating pyrimidine and triphenylamine fragments were described. This oligomer exhibits very high two photon absorption ability and was efficiently employed as photosensitizer for two photon lithography.

\section{ABSTRACT.}

A comparative study of the linear and nonlinear optical properties of a novel triphenylaminepyrimidine alternated oligomer and its corresponding $\mathrm{V}$-shaped quadrupolar monomer is presented. Both chromophores strikingly exhibit the same spectral shape when considering their respective one- and two-photon absorption spectra. This effect was attributed to a weak 
interchromophore coupling within the oligomer which exhibits a highly distorted geometry resulting in a strong reduction of the effective conjugation length. The recursive implementation of nine monomers into a three-dimensional architecture leads however to a cooperative enhancement of the two-photon absorption (2PA) cross-section with a $\delta_{\mathrm{MAX}}$ of $5093 \mathrm{GM}$ at $800 \mathrm{~nm}$. This very high 2PA ability has been oriented to improve the two-photon induced polymerization efficiency of a bicomponent photoinitiator system implying a hexaarylbiimidazole used as $\mathrm{H}$-abstractor and an aliphatic amine used as H-donor. The photosensitizing mechanism is investigated and we clearly show that the intrinsic photoinitiation efficiency with the oligomer is increased by a factor 3 as compared to its corresponding monomer. We therefore demonstrate that such a two-photon sensitizing strategy leads to a synergy effect combining a higher photoinitiation reactivity and a very large two-photon absorption cross-section. 


\section{INTRODUCTION}

Two-photon absorption (2PA) materials are of primary importance in a wide range of promising applications such as three-dimensional optical storage memory, ${ }^{1}$ photodynamic therapy, ${ }^{2}$ high-resolution imaging ${ }^{3}$ or multiphoton stereolithography. ${ }^{4}$ In the case of twophoton polymerization (2PP), a tight confinement of the excitation volume around the focal point is obtained allowing the fabrication of intricate 3D structures with feature sizes as small as $100 \mathrm{~nm} .^{1 \mathrm{c}, 4 \mathrm{a}}$ The $2 \mathrm{PP}$ technology also implies the use of photoactivable systems with both large 2PA cross sections $(\delta)$ and high photoinitiating reactivity. Despite their very low 2PA cross-sections, ${ }^{5}$ commercially available photoinitiators can be successfully employed for twophoton polymerization when associated with two-photon activable sensitizers. ${ }^{6}$ From a practical point of view, such a bi-component approach constitutes one of the simplest and reliable processing methods since it takes benefit of the very high reactivity of well known UV-curing initiators ${ }^{7}$ and of advances in engineering researches for the design of chromophores with large $2 \mathrm{PA}$ cross-section. ${ }^{3 \mathrm{a}-\mathrm{b}, 8}$ In this domain, several strategies ${ }^{8-9}$ have been proposed to establish some relevant structure/property relationships to enhance $\delta$. In the so-called 'multi-branching' approach, ${ }^{\text {9b,9e-f,10 }}$ various electron-accepting and -donating groups can be implemented within the core or at the periphery of multibranched $\pi$-conjugated structures used as 'electron relays'. The nature of donor and acceptor substituents, ${ }^{8}$ the $\pi$ delocalization length, ${ }^{9 c, 11}$ the strength of the interbranch coupling, ${ }^{9 b, 9 e-f}$ the spatial conformation and the electronic symmetry ${ }^{12}$ of the global architecture ${ }^{9 a, 13}$ are all distinctive parameters that can be methodically tuned to optimize the 2PA ability of the final chromophore. In this context, $\pi$-conjugated oligomers and $\pi$-conjugated polymers constitute the typical molecular platform which can rationalize such fundamental issues. ${ }^{14}$ In this recursive synthesis approach, the generation of $\pi$-conjugated macromolecules can offer a very high degree of electronic delocalization resulting in a strong enhancement of the two-photon absorption cross section. ${ }^{14 \mathrm{~b}-\mathrm{d}, 15}$ For instance, Williams-Harry et al. ${ }^{16}$ have elaborated a series of macrocyclic thiophene $n$-mers integrating more than twelve thienylene units which exhibit giant 2PA cross sections up to $11 \times 10^{5} \mathrm{GM}\left(1 \mathrm{GM}=10^{-50} \mathrm{~cm}^{4} \mathrm{~s}\right.$ photon $\left.{ }^{-1}\right)$. In the same manner, Yoon et al. ${ }^{17}$ have synthesized fused $\mathrm{Ni}^{2+}$ porphyrin arrays containing up to 5 macrocycles leading to a $\delta_{\text {MAX }}$ of about $4.1 \times 10^{5} \mathrm{GM}$. In this latter example, the $\pi$-conjugated chains with enforced coplanarity constitute the good guarantee for a great effective conjugation length ${ }^{14 a}$ with a maximizing effect of the $2 \mathrm{PA}$ cross-section. The dendrimer 
architecture $^{18}$ also offers the possibility of increasing the density of effective chromophores. Even though such a highly ordered confined geometry can potentially disrupt the conjugation, ${ }^{12 \mathrm{a}, \mathrm{b}}$ the strong interchromophore coupling can promote a cooperative enhancement of the nonlinear optical response. For instance, Varnavski et al. ${ }^{12 \mathrm{c}}$ have reported the photophysical characterization of tolane-based dendritic chromophores which undergo a strong enhancement of $\delta$ over the additive behaviour when going from the trimer derivative (9 chromophores) to higher generations (up to 21 chromophores).

In this contribution, we report the synthesis and the photophysical characterization of a new $\pi$-conjugated oligomer alternating triphenylamine (D) and pyrimidine (A) moieties connected with vinylene $(-\pi-)$ bridges (Scheme 1). Based on a large set of experimental results such as absorption, emission, excitation anisotropy or 2PA spectra associated with theoretical calculations, a comparison of the linear and the nonlinear optical properties of the oligomer $(\mathbf{O g})$ and its D- $\pi$-A- $\pi$-D model chromophore $(\mathbf{M})$ is performed in order to highlight the role of dimensionality as well as the strength of interchromophore coupling. Finally we will take benefit of the very large 2PA ability of $\mathbf{O g}$ to demonstrate the potential interest of this new oligomer as efficient photosensitizer for one and two-photon polymerization applications.<smiles>C(=C/c1cc(/C=C/c2ccc(N(c3ccccc3)c3ccccc3)cc2)ncn1)\c1ccc(N(c2ccccc2)c2ccccc2)cc1</smiles>

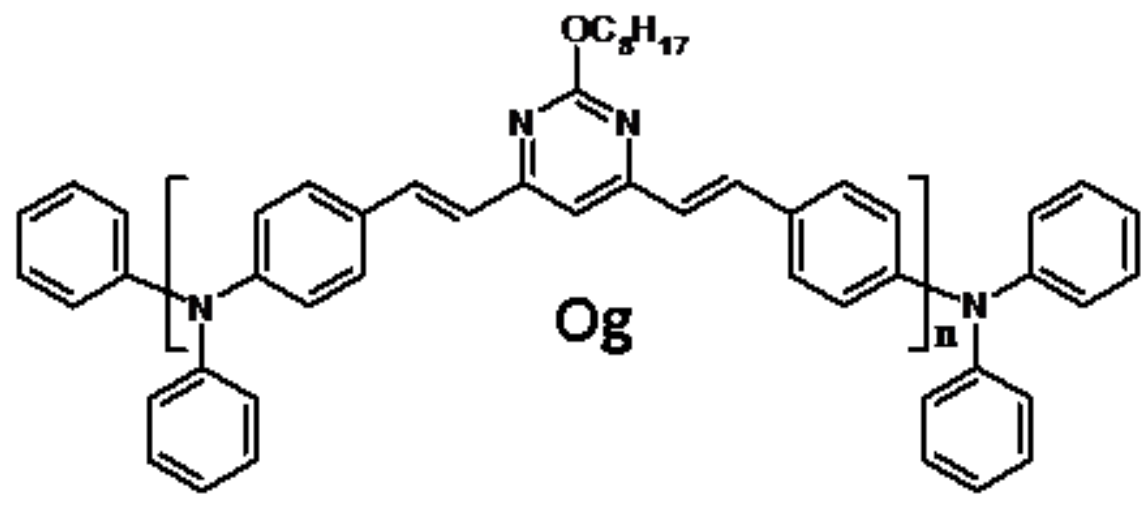

Scheme 1. Molecular structures of chromophores. 


\section{RESULTS AND DISCUSSION}

\subsection{Synthesis.}

The synthesis and full characterization of the model chromophore $\mathbf{M}$ has been previously reported by some of us. ${ }^{19}$ In order to obtain an oligomer with enough solubility, a long alkoxy chain was added to the pyrimidine heterocycle. Starting from commercially available 2chloro-4,6-dimethylpyrimidine, a nucleophilic substitution with lithium octylate was carried out. The desired product was obtained with good yield (Scheme 2). The polymerization was carried out by condensation reaction between dimethylpyrimidine derivative 3 and 4,4'diformyltriphenylamine 4 in boiling aqueous $5 \mathrm{M} \mathrm{NaOH}$ using aliquat ${ }^{\circledR} 336$ as a phase transfer catalyst according to the method initially described by Vanden Eynde. ${ }^{20}$ Indeed it has been shown that such condensation of methylpyrimidine derivatives with aldehyde is complete. The non quantitative yield sometimes obtained is mainly due to substantial loss during the purification process of the compounds. ${ }^{19,21}$ The desired oligomer was obtained in good yield after purification by precipitation. Other conditions were tested using potassium tertbutoxyde in THF, but in that case a material which was completely insoluble in common organic solvent was obtained. The oligomer has been analyzed by NMR spectrometry, the ${ }^{1} \mathrm{H}$ spectrum is given in supporting information (Figure S1). The two doublets that are observed at 7.85 and $6.91 \mathrm{ppm}$ with a coupling constant of $15.5 \mathrm{~Hz}$ are consistent with an oligomer having double bonds with an $E$ configuration. No signal corresponding to an aldehyde function is observed but a singlet at $2.44 \mathrm{ppm}$ corresponds to methyl groups borne by a pyrimidine ring indicating the nature of the end groups of the oligomer. Comparison of the integration of this signal with the integration of the signal of one of the hydrogen of the double bond at $7.85 \mathrm{ppm}$ indicates an average number of repeat motifs of 13.6, which corresponds to an average molecular weight of around 7000 g.mol ${ }^{-1}$. Size exclusion chromatography analysis were also carried out giving a number-average molecular weight $M_{n}$ $=4650 \mathrm{~g} \cdot \mathrm{mol}^{-1}$ and a polydispersity indice PDI $=1.63\left(M_{w}=7200 \mathrm{~g} \cdot \mathrm{mol}^{-1}\right)$. Therefore the oligomer $\mathbf{O g}$ corresponds to a recursive association of around 8 to $10 \mathrm{M}$-units (see Scheme 1). Hereafter, we will take an average value of 9 M-units for the oligomer. This oligomer has a good solubility in various solvents such as chloroform, dichloromethane or tetrahydrofuran. It does not show any self-aggregation as confirmed by checking Beer Lambert law within mM 
concentration range. Finally the X-ray diffraction spectrum of the powder of $\mathbf{O g}$ (Figure S2) exhibits a broad peak at $2 \theta=19.5^{\circ}$ which suggests an amorphous form for this oligomer.

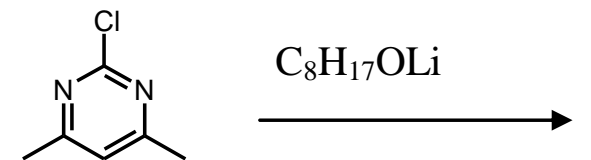

THF, $\Delta, 15 \mathrm{H}$ $67 \%$<smiles>COc1nc(C)cc(C)n1</smiles>

3

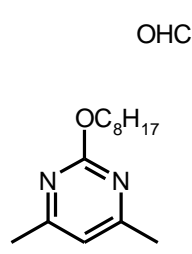

3

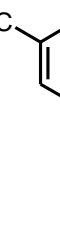<smiles>CC(C)N(c1ccccc1)c1ccccc1</smiles>

4

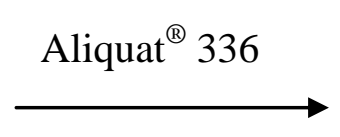

$\mathrm{NaOH} 5 \mathrm{M}, \Delta, 2 \mathrm{H}$ $94 \%$<smiles>COc1nc(/C=C/c2ccc(N(c3ccccc3)c3ccccc3)cc2)cc(/C=C/c2ccc(N(c3ccccc3)c3ccccc3)cc2)n1</smiles>

Scheme 2. Reaction scheme for the synthesis of the oligomer $\mathbf{O g}$.

\subsection{Geometry and Electronic Properties.}

The fully optimized structure of the model chromophore calculated at DFT level is presented in Figure 1A. The core of the dye which corresponds to a 4,6-bis(styryl)pyrimidine subunit adopts a quasi-planar conformation. The sterical hindrance around the external nitrogen atoms leads to a twist angle of about $\sim 34^{\circ}$ around the $\mathrm{C}-\mathrm{N}$ bonds connecting the diphenylamine and styryl moieties. Moreover all external phenyls are tilted of about $\sim 65^{\circ}$ with respect to the central plane. Therefore the geometry of $\mathbf{M}$ can be presented as a successive alternation of planar and highly twisted domains. Such a structural alternation can be generalized when extending the molecular dimensionality. For instance, Figure 1B displays the fully optimized structure of an oligomer associating three M-units. Note that methoxy substituents have been implemented in position 2 of each pyrimidine to demonstrate that this substituent hardly influences the planarity of the 4,6-bis(styryl)pyrimidine fragments. The oligomer clearly exhibits a helical shaped geometry which globally preserves the structural characteristics of each repeat motif: $i$ ) a quasi-planar 4,6-bis(styryl)pyrimidine subunit $i i$ ) the interplane angles between two adjacent fragments have an average value of $\sim 63^{\circ} \mathrm{iii}$ ) the twist angles of the connecting $\mathrm{C}-\mathrm{N}$ bonds slightly increases by about $3^{\circ}$. Hence increasing the number of repeat 
units leads to a strongly distorted conformation that should drastically limit the extent of electronic delocalization all along the $\pi$-conjugated backbone. Figure $\mathbf{2}$ displays the normalized absorption and fluorescence spectra of $\mathbf{M}$ and $\mathbf{O g}$ in dichloromethane along with their respective steady-state excitation anisotropies. Table 1 gathers the corresponding spectroscopic data. The calculated absorption spectrum of $\mathbf{M}$ using TD-DFT method is also presented on the top of the experimental spectrum and Table 2 reports all related calculated parameters. The low energy part of the absorption spectrum of $\mathbf{M}$ is dominated by an intensive and unstructured band which shows a very slight shoulder at its blue edge (i.e. around $410 \mathrm{~nm}$ ). Interestingly the anisotropy remains constant within this long wavelength absorption band but strongly decreases below $420 \mathrm{~nm}$ and reaches a plateau below $375 \mathrm{~nm}$. Such a change in anisotropy is clearly corroborated by TD-DFT calculations which indicate the presence of two well separated electronic transitions with distinctive polarization (see Table 2). The calculated energy of the $S_{0} \rightarrow S_{1}$ transition reasonably agrees with the experimental value of the 0-0 transition energy $\left(\mathrm{E}_{00}\right)$. This latter energy was measured from the intercept of the normalized absorption and fluorescence spectra recorded in apolar medium (hexane) in order to minimize the solvent induced relaxation process at $S_{1}$ state. In this condition, a mirror-image relationship is observed between absorption and fluorescence spectra which leads to a more reliable value for $\mathrm{E}_{00}$ (Figure S3). According to our TD-DFT calculations, the lowest energy transition is a pure HOMO-LUMO transition which is strongly one-photon allowed $(f>1)$ and polarized along the long axis of the molecule ( $x$-axis in Figure 1A). The $S_{0} \rightarrow S_{2}$ transition is weakly allowed and corresponds to a pure HOMO${ }_{-1}$ LUMO transition. It is interesting to note that the polarization of this $S_{0} \rightarrow S_{2}$ transition is perpendicular to that of the $\mathrm{S}_{0} \rightarrow \mathrm{S}_{1}$ one which is in line with the strong change in anisotropy within this spectral region. Moreover, it should be noted that the average value for the anisotropy of the $S_{0} \rightarrow S_{1}$ is about $0.25 \pm 0.01$. This indicates that the transition moment at the emitting state is not collinear to that of the ground state. Such a change in the orientation of the absorption and emission moments should be presumably ascribed to a photoinduced planarization affecting the triphenylamines at the relaxed $S_{1}$ state. Such a relaxation effect has been previously observed for the excited-states geometry of multibranched D- $\pi$-A dipoles. ${ }^{6 \mathrm{~d}-\mathrm{e}}$ In the 315-370 nm range (see Figure 2), the anisotropy remains constant due to the equivalent polarizations of the dominant transitions $\mathrm{S}_{0} \rightarrow \mathrm{S}_{3}$ and $\mathrm{S}_{0} \rightarrow \mathrm{S}_{5}$ respectively. The electronic distributions of the frontier orbitals are shown in Figure 3. The two lowest energy transitions clearly correspond to $\pi-\pi^{*}$ transitions implying a charge transfer (CT) from the periphery 
(triphenylamino group) to the core (pyrimidine ring) of the dye. Interestingly, the occurrence of these two CT transitions can be predicted within the framework of the Frenkel exciton model. ${ }^{\text {a-b,22 }}$ For a two-branched V-shaped chromophore, it is assumed that interbranch electrostatic interactions lead to the splitting of the one-exciton transition relative to the molecular synthon as illustrated in Scheme 3. Moreover for a homo-dimeric chromophore, this twofold degeneracy occurs symmetrically and leads to a $S_{1}-S_{2}$ gap of $2 \mathrm{~V}$ where $\mathrm{V}$ denotes the interbranch coupling. In our case and according to our TD-DFT calculations, the interbranch coupling which corresponds to half value of the $S_{1}-S_{2}$ energy gap (see Scheme 3 ) can be theoretically estimated to ca. $0.13 \mathrm{eV}$. Such a value is comparable to those obtained from other stilbenyl quadrupolar chromophores symmetrically substituted with amino end groups. ${ }^{9 \mathrm{~b}, 9 \mathrm{e}-\mathrm{f}}$ It is also noteworthy that this interbranching coupling is noticeably stronger than that calculated for a similar V-shaped structure with the same 4,6-bis(styryl)pyrimidine core but associating moderate pyran-based donors at the periphery. ${ }^{23}$

Table 1. Summary of optical data and parameters for $\mathbf{M}$ and $\mathbf{O g}$.

\begin{tabular}{cccccccccc}
\hline & $\begin{array}{c}\lambda_{a b s} \\
/ \mathrm{nm}\end{array}$ & $\begin{array}{c}\varepsilon^{M A X} \\
/ 10^{3} \mathrm{M}^{-1} \mathrm{~cm}^{-1}\end{array}$ & $\begin{array}{c}\lambda_{\text {fluo }} \\
/ \mathrm{nm}\end{array}$ & $\Phi_{\text {fluo }}$ & $\begin{array}{c}\tau_{\text {fluo }} \\
/ \mathrm{ns}\end{array}$ & $\begin{array}{c}\mathrm{k}_{r}^{b} \\
/ 10^{8} \mathrm{~s}^{-1}\end{array}$ & $\begin{array}{c}\delta_{\text {MAX }}\left(\lambda_{\text {MAX }}\right) \\
/ \mathrm{GM}\end{array}$ & $\mathrm{N}_{\text {eff }}$ & $\delta / \mathrm{N}^{2}$ eff \\
\cline { 2 - 9 } $\mathbf{M}$ & 431 & 56.5 & 554 & 0.82 & 2.90 & 2.8 & $360(800 \mathrm{~nm})$ & 25 & 0.58 \\
$\mathbf{O g}$ & 449 & 549.6 & 558 & 0.63 & $2.60{ }^{a}$ & 2.4 & $5093(800 \mathrm{~nm})$ & 69 & 1.07 \\
\hline
\end{tabular}

Table 2 TDDFT and experimental electronic transitions of $\mathbf{M}$.

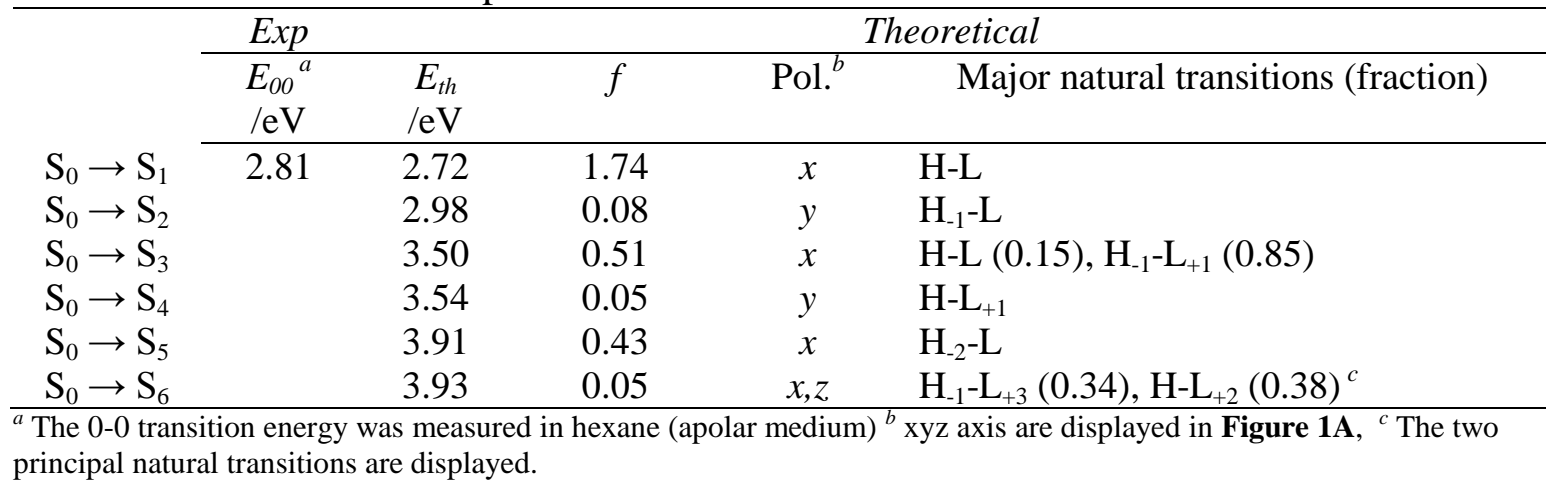




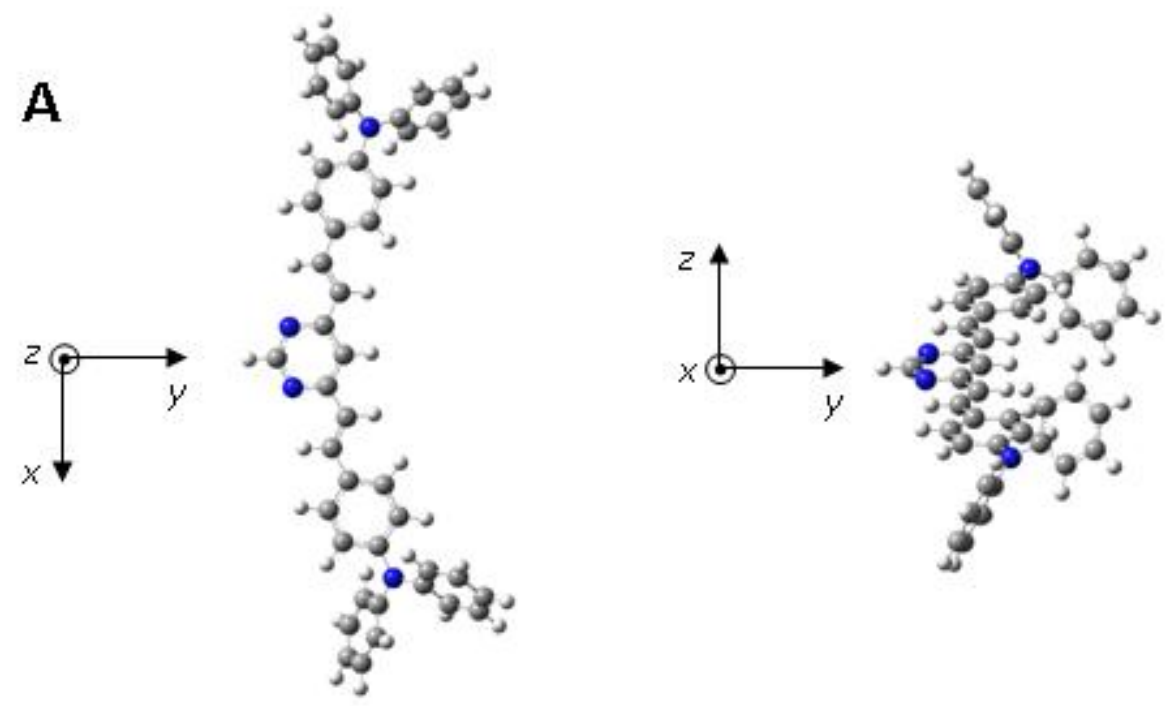

B
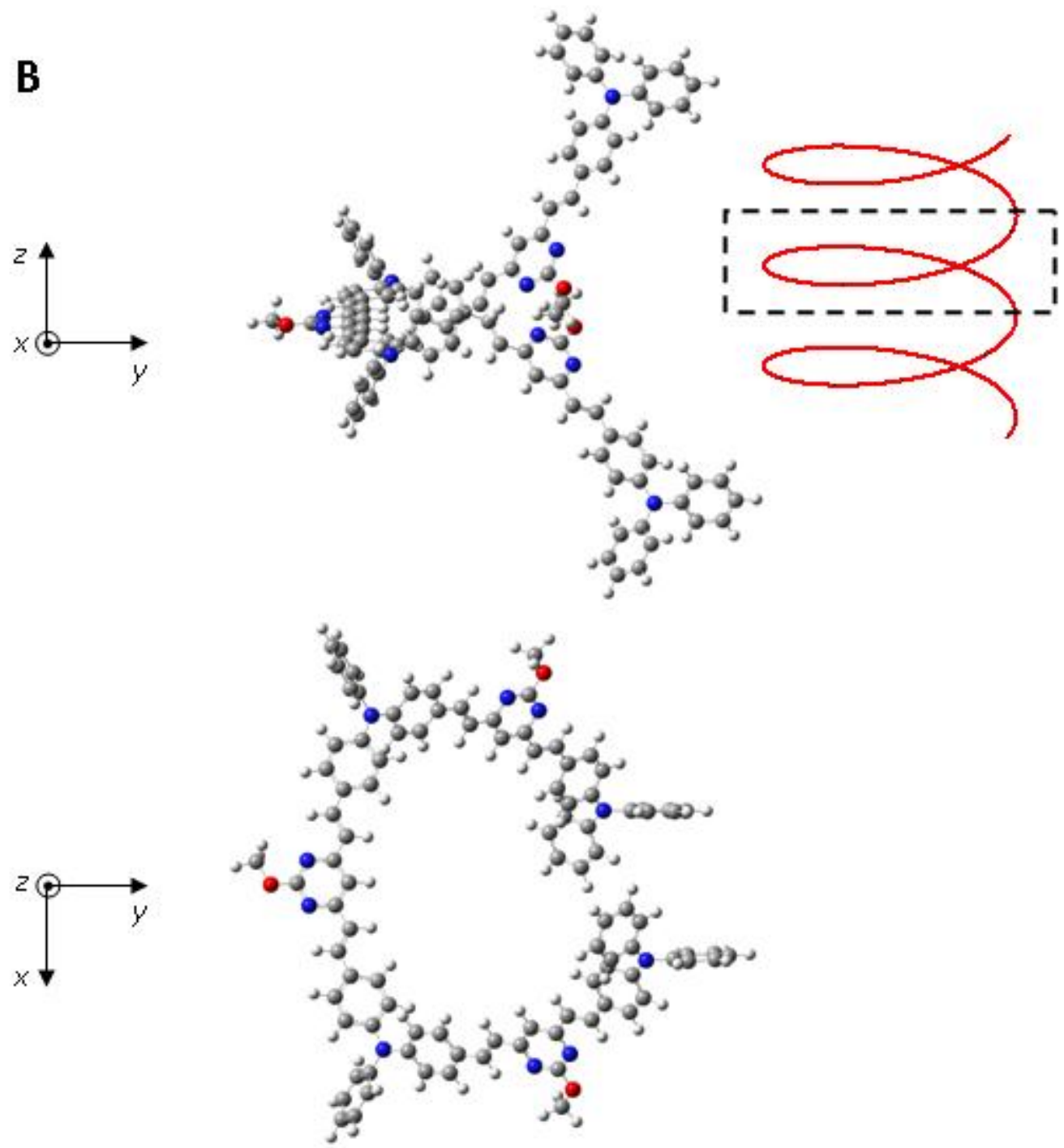

Figure 1. Optimized geometries of $\mathbf{M}$ and its oligomeric model with three $\mathbf{M}$-units (PBE0/6-31G(d) level). 

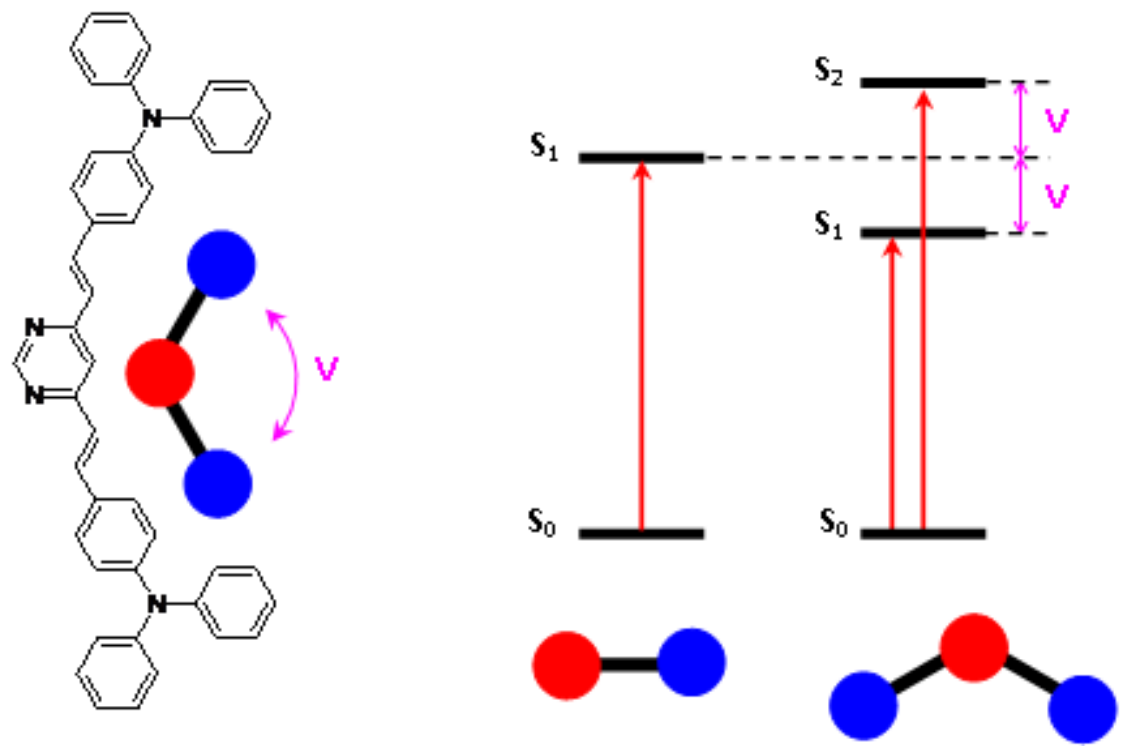

Scheme 3. Schematic electronic level diagrams within the excitonic coupling model for a dipolar unit and its corresponding V-shaped quadrupolar structure.

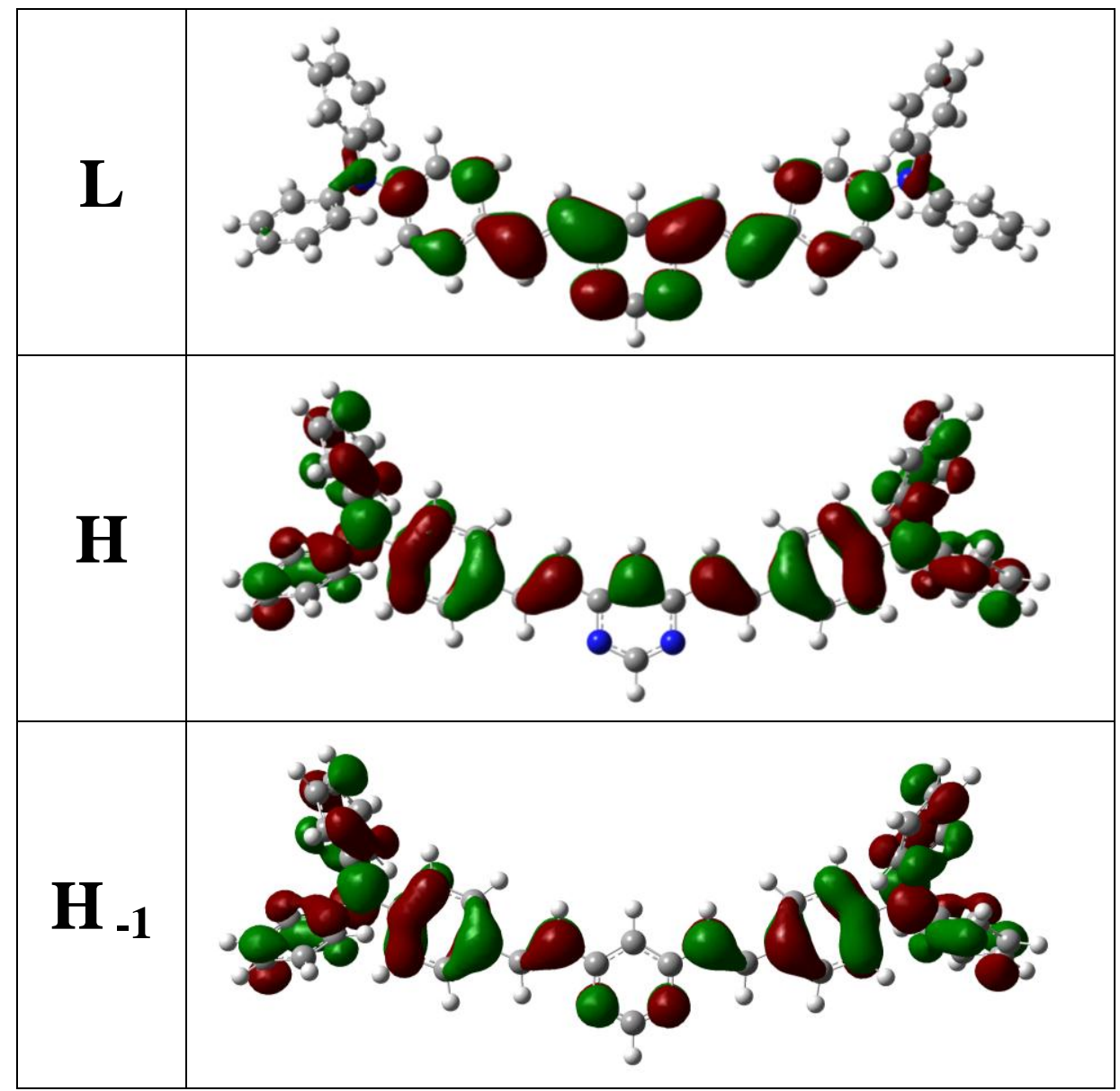

Figure 3. Representation of the molecular orbitals involved in the $S_{0} \rightarrow S_{1}$ and $S_{0} \rightarrow S_{2}$ electronic transitions of $\mathbf{M}$. 
Surprisingly, increasing the number of repeat units (from $n=1$ to 9 ) leads to a very strong hyperchromic effect with a 10-fold increase of $\varepsilon_{\text {MAX }}$ (Table 1) but hardly affects the shape of the absorption spectrum. Indeed, on going from $\mathbf{M}$ to $\mathbf{O g}$, the longest wavelength absorption band undergoes a slight broadening (x 1.2) and is hardly redshifted by about 930 $\mathrm{cm}^{-1}$. This very weak bathochromism contrasts, for instance, with that observed for oligo( $p$ phenylvinylene $) \mathrm{s}^{14 \mathrm{e}}$ or oligo( $\alpha$-thiophene) $\mathrm{s}^{14 \mathrm{c}-\mathrm{d}, 24}$ which exhibit a band redshift of about $8000 \mathrm{~cm}^{-1}$ upon increasing the number of repeat units up to 12-mers. Contrary to these relatively planar oligomers, the severe steric interactions between each adjacent $\mathbf{M}$-unit result in a serious disturbance of the $\pi$-orbital overlap along the oligomer backbone. Such similar structural effects have been nicely rationalized by the working groups of Osuka and Kim ${ }^{25}$ who have elaborated two series of porphyrin-based oligomers ( $n=2$ to 12) which show respectively a planar and an alternatively twisted geometry. Whereas the planar fused porphyrin series display strongly red-shifted absorption spectra upon increasing the size of the oligomer ( $\Delta v \sim 6100 \mathrm{~cm}^{-1}$ for $n=2$ to 12$)$, the twisted series undergo a 5-fold weaker redshift. This structure-property relationship is clearly in line with the effects observed in our case and presumably confirms the highly distorted structure of our oligomer. It turns out that the effective conjugation length ${ }^{14 a}$ within our D- $\pi$-A alternating oligomer is reached very fast upon increasing the number of repeat units. As shown in Figure 2, both $\mathbf{M}$ and $\mathbf{O g}$ exhibit a second distinctive band near $300 \mathrm{~nm}$. This band is usually observed for $\mathrm{N}$-phenylated systems ${ }^{10,26}$ and should be ascribed to the $\mathrm{S}_{0} \rightarrow{ }^{1} \mathrm{~L}_{a}$ transitions ${ }^{27}$ localized on the triphenylamine moieties. ${ }^{28}$ Interestingly, the intensity of this band relative to the lowest energy one only decreases from 0.68 to 0.50 on going from $\mathbf{M}$ to $\mathbf{O g}$. Such a slight effect illustrates a weak mixing of the localized ${ }^{1} \mathrm{~L}_{\mathrm{a}}$ states with the low lying ${ }^{1} \mathrm{CT}$ ones ${ }^{29}$ and corroborates the assumption that $\mathbf{O g}$ can be viewed as a composite macromolecule comprising molecular units which preserve their electronic individuality. According to the point-dipole exciton model proposed by Kasha et al. ${ }^{30}$ the coupling between consecutive Munits should lead to a resonance splitting of the excited state composite molecule energy levels which were initially degenerate. For a strong exciton coupling, the splitting energy should give rise to a clear separation of the new $S_{0} \rightarrow S_{n}$ transitions. However, in our case, a slight band broadening (x 1.2) is only observed and the corresponding anisotropy spectrum of Og displays globally the same changes as those observed for M (Figure 2). Such a weak interchromophore exciton coupling is also corroborated by the equivalent fluorescence bands 
of both dyes. Moreover, for the oligomer, it should be indicated that the population of emitting conformers is clearly monodispersive since the fluorescence band shape of $\mathbf{O g}$ does not show any excitation wavelength dependencies and the corresponding excitation spectra collected at various emission wavelengths match perfectly the absorption spectrum. The fluorescence decay of $\mathbf{O g}$ can be confidently fitted with two exponential functions but exhibits a major long-lived component whose contribution is larger than $95 \%$ of the total emission (see Figure S4). Even though the fluorescence quantum yield $\left(\Phi_{\text {fluo }}\right)$ decreases on going from $\mathbf{M}$ to $\mathbf{O g}$ (see Table 1), the oligomer remains highly emissive with $\Phi_{\text {fluo }}=0.63$ in dichloromethane. The radiative rate constant $\left(\mathrm{k}_{r}\right)$ of $\mathbf{O g}$ is comparable to that calculated for $\mathbf{M}$ which indicates an electronic equivalency of the two emitting states and therefore confirms that the radiative deactivation of $\mathbf{O g}$ presumably stems from a localized excited state centered a single M-unit. This electronic localization we proposed for the emitting state of $\mathbf{O g}$ is also in line with the significant change in the orientation of the absorption and emission moments as indicated by the relatively low value of the $S_{0} \rightarrow S_{1}$ emission anisotropy which exhibits an average value of $c a$. $0.14 \pm 0.02$.

Hence the oligomer $\mathbf{O g}$ clearly exhibits a similar photophysical fingerprint as that observed for its model chromophore M. This electronic effect should be attributed to a highly distorted geometry which dramatically reduces the effective conjugation length (ECL) of this donor-acceptor $\pi$-conjugated oligomer.

\subsection{Two-photon Absorption (2PA) Properties.}

The equivalency observed for the electronic properties of $\mathbf{M}$ and $\mathbf{O g}$ can be extended to the shape of their respective two-photon absorption (2PA) spectra. Such an effect has been previously reported by Drobizhev et al. ${ }^{12 \mathrm{a}-\mathrm{b}}$ when comparing the $2 \mathrm{PA}$ spectra of the 4,4'bis(diphenylamino)stilbene and several generations of dendrimers elaborated through recursive association of this latter molecular building block. Here also, the successive alternation of planar and highly distorted domains should result in a loss of conjugation all long the macromolecular structure. Figure 4 shows the 2PA spectra of our compounds. The two-photon absorption cross-sections $(\delta)$ have been measured in the $680-1000 \mathrm{~nm}$ range by means of two-photon excited fluorescence. ${ }^{31}$ The 2PA bands are plotted against half the excitation wavelength to have a direct comparison with the one-photon absorption (1PA) spectra. According to our spectral resolution, both spectra present two distinctive bands 
localized in the same spectral range as the longest wavelength 1PA band. Their respective maximum absorption wavelengths (i.e. $\lambda_{2 \mathrm{PA}} \approx 900 \mathrm{~nm}$ and $800 \mathrm{~nm}$ ) nicely correspond to the position of the $S_{0} \rightarrow S_{1}$ and $S_{0} \rightarrow S_{2}$ transitions. Moreover one clearly observes that the highenergy band is more intense than the low-energy one. This effect should be related to the dipole selection rule for nearly centrosymmetric molecular symmetry which brings the $S_{1}$ state to be almost 2PA forbidden whereas the $S_{2}$ becomes 2PA allowed. Even though this trend is globally observed it is clear that the $S_{0} \rightarrow S_{1}$ transition becomes partially allowed for both compounds probably due to a relaxation of the molecular structures to geometries with a lower symmetry. The most important effect observed upon increasing the dimensionality is the very strong enhancement of $\delta_{\text {MAX }}$ which increases from 360 GM to 5093 GM on going from $\mathrm{M}$ and $\mathrm{Og}$. Therefore the value of $\delta_{\mathrm{MAX}}$ per repeat unit is about $566 \mathrm{GM}(n \sim 9)$ which clearly indicates a cooperative enhancement of the 2PA properties of the oligomer. However it should be emphasized that $\delta_{\text {MAX }}$ depends quadratically on the effective number of electrons $\left(\mathrm{N}_{\text {eff }}\right)$ which contribute to the non linear response of the molecule. ${ }^{32}$ This parameter can be determined according to the counting method proposed by Kuzyk et al. ${ }^{32}$ Therefore, when normalizing the 2PA cross-section by the square of the effective number of electrons $\left(\delta / \mathrm{N}^{2}\right.$ eff $)$, a direct comparison can be made between the intrinsic 2PA properties within a series of chromophores. This methodology allows a better quantification of the cooperative enhancement or weakening of the nonlinear optical response of the macromolecular structure. According to this normalization approach, a moderate cooperative effect is observed with a 1.8-fold increase of the intrinsic cross-section of $\mathbf{O g}$ as compared to $\mathbf{M}$. This enhancement is comparable to the 1.4-fold increase of $\delta / \mathrm{N}^{2}$ eff on going from the 4,4'-bis (diphenylamino)stilbene $\left(\delta_{\mathrm{MAX}}=320 \mathrm{GM}, \mathrm{N}_{\text {eff }}=18\right)$ to its second-order generation dendrimer with 13 repeat units ${ }^{12 \mathrm{a}}\left(\delta_{\mathrm{MAX}}=4500 \mathrm{GM}, \mathrm{N}_{\text {eff }}=56\right)$. It is worth stressing that the strategy consisting in increasing the dimensionality of a macromolecule does not lead necessary to a significant improvement of its intrinsic 2PA performance. For instance the working groups of Goodson and Iyoda ${ }^{16,33}$ have studied the 2PA properties of several very large macrocyclic thiophene $n$-mers comprising up to 30 thienylene-acetylene repeat units. In this precise case, the optimal value for $\delta / \mathrm{N}^{2}$ eff was observed for the derivative with 18 -mers $\left(\delta / \mathrm{N}_{\text {eff }}^{2} \sim 3.2\right)$ whereas the largest one $\left(\delta_{\mathrm{MAX}}=107800 \mathrm{GM}, \mathrm{N}_{\text {eff }}=240\right)$ clearly leads to a decrease of $\delta / \mathrm{N}^{2}$ eff by a factor 1.7 with respect to the optimal structure. From a conceptual point of view, it is clear that the intrinsic performance of a macromolecule can not be scaled through several orders of magnitude but from a practical approach a very large enhancement 
of $\delta_{\text {MAX }}$ undoubtedly constitutes the guarantee to efficiently promote any two-photon activation processes.
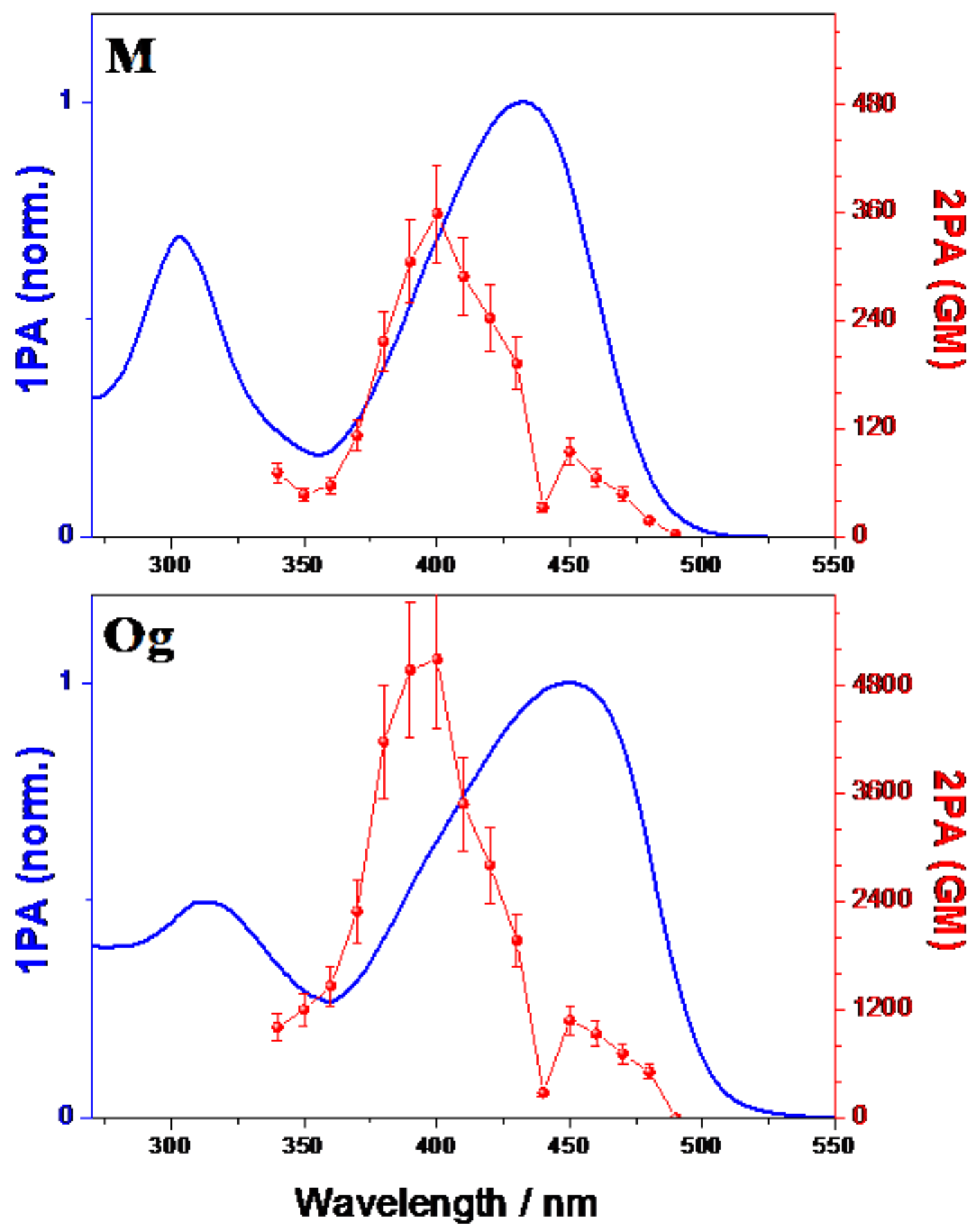

Figure 4. 1PA (full lines) and 2PA spectra (plotted against half of the excitation wavelenghth) of chromophores in dichloromethane. 
Therefore, in order to take benefit of the very large 2PA cross-section of $\mathbf{O g}$, we will demonstrate hereafter that this chromophore can be used as an efficient two-photon sensitizer for multiphoton stereolithography applications.

\subsection{One- and Two-photon Sensitized Polymerization.}

Figure 5 shows the FT-RTIR (i.e. Fourier transform real-time infrared) kinetic curves during visible irradiation of four distinctive diacrylate formulations which all contains a hexaarylbiimidazole derivative (HABI) (Scheme 4). This chromophore is a one of the most widely patented free-radical UV-Visible photoinitiator. ${ }^{34}$ Upon direct excitation, ${ }^{35}$ HABI undergoes a homolytic cleavage resulting in the formation of two lyophyl radicals $(\mathbf{L} \bullet)^{36}$ as shown in Scheme 4. However this latter radical adds very slowly to acrylic monomer in such manner that HABI is intrinsically a very poor free radical photoinitiator as illustrated in Figure 5. Moreover, it should be indicated that the photopolymerization is performed at 440 $\mathrm{nm}$ irradiation which corresponds to the extreme red edge of the absorption spectrum of HABI (inset Figure 5). Therefore HABI is usually associated with a hydrogen donor coinitiator. $^{34 b, 37}$ For instance, in presence of N-methyl diethanolamine (MDEA), the photopolymerization is significantly improved with $28 \%$ conversion of acrylate functions after $150 \mathrm{~s}$ of irradiation. In this case, the mechanism is somewhat different since a H-transfer reaction occurs from MDEA to $\mathbf{L} \bullet$ which generates a $\alpha$-aminoalkyl radical. This latter species exhibits a very high reactivity upon addition reactions ${ }^{38}$ onto double bonds of acrylate monomers. The sequential steps for the generation of primary radicals can be described as follows:

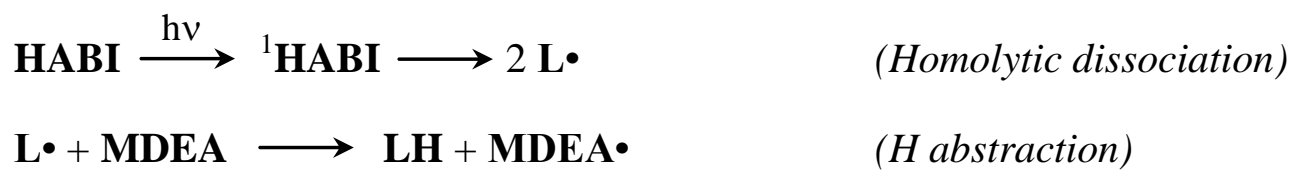




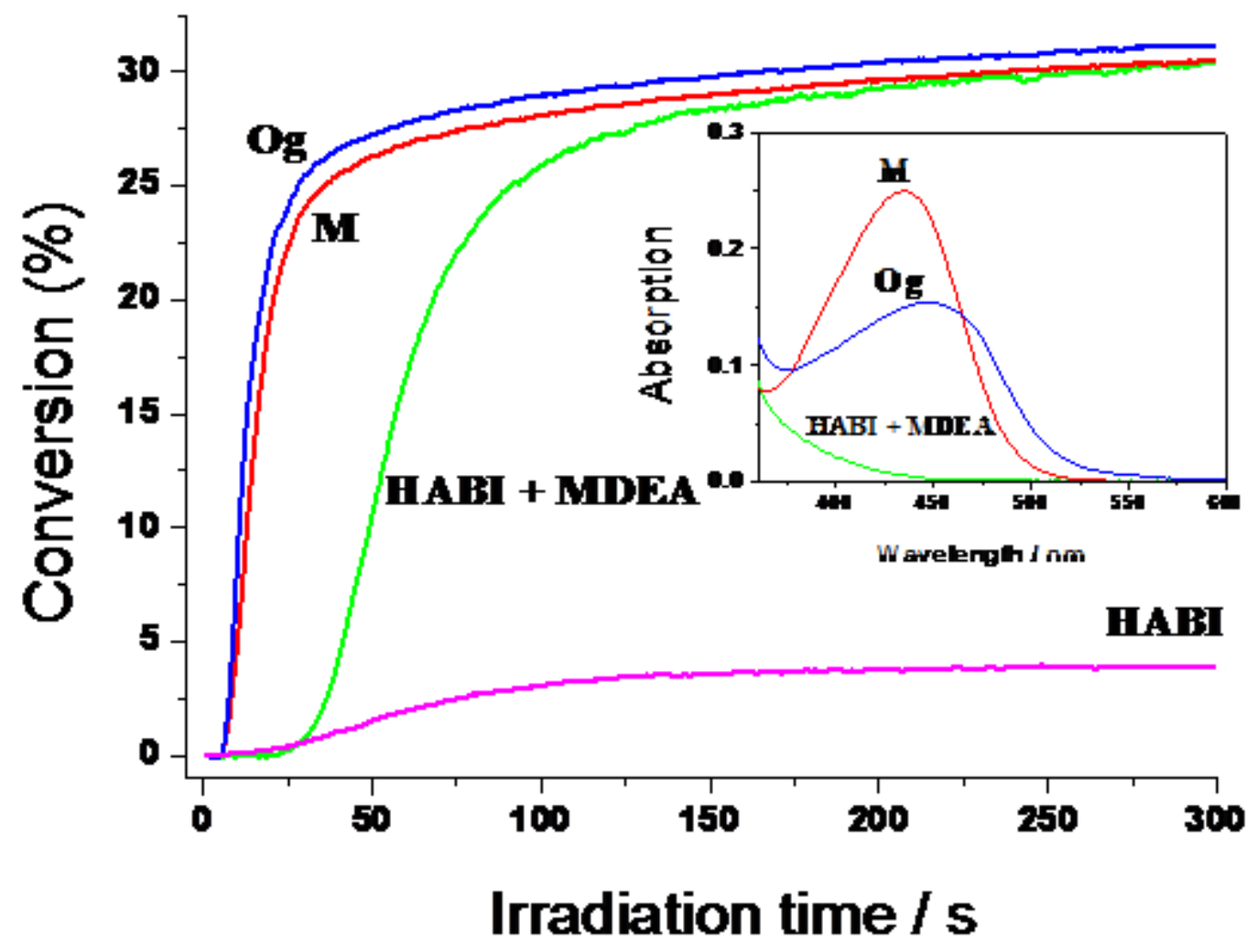

Figure 5. Real-time FTIR kinetics conversion curves for visible-curing at $440 \mathrm{~nm}$ of diacrylate resins with 'HABI' : HABI : 1.5 wt \%, 'HABI+MDEA' : HABI / MDEA : $1.5 \mathrm{wt} \% / 3 \mathrm{wt} \%$, ' $\boldsymbol{M}$ ' HABI / MDEA / M : $1.5 \mathrm{wt} \% / 3 \mathrm{wt} \%$ / 0.12 wt $\%$, ' $\boldsymbol{O g}$ ' : HABI / MDEA / Og : 1.5 wt $\% / 3$ wt $\% / 0.07$ wt $\%$.

Inset : Absorption spectra of the corresponding diacrylate resins $(25-\mu \mathrm{m}$-thick films).

However such a reducible radical ${ }^{39}$ is also sensitive to the presence oxygen dissolved in monomer medium. This inhibition process is typically illustrated on the kinetic profile by the long induction time ( $25 \mathrm{~s}$ ) observed during the first step of photopolymerization. It corresponds to the time necessary for the total consumption of the dissolved oxygen. Interestingly, the presence of photosensitizers (PS) drastically reduces this inhibition time by a factor 6 and leads to a strong increase of the polymerization rates which are multiplied by a factor 2.9 for $\mathbf{M}$ and 4.4 for $\mathbf{O g}$. This clear improvement of the photoinitiation should be connected to the high absorptivity of $\mathbf{M}$ and $\mathbf{O g}$ at the irradiation wavelength (see inset of Figure 5) which leads to a larger amount of primary excited species. The photosensitizing process in presence of PS typically corresponds to a sequential reaction ${ }^{34 b, 35 b, 37 b-c, 40}$ starting from a photoinduced electron transfer from the singlet excited state of PS $\left({ }^{1} \mathbf{P S}\right)$ to HABI 
which first generates the radical cation $\mathbf{P S}^{{ }^{++}}$and the radical anion $\mathbf{H A B I}{ }^{{ }^{-}}$. This latter radical consecutively undergoes a cleavage to produce the neutral free radical $\mathbf{L} \bullet$ and its associated radical anion $\mathbf{L}^{--}$:

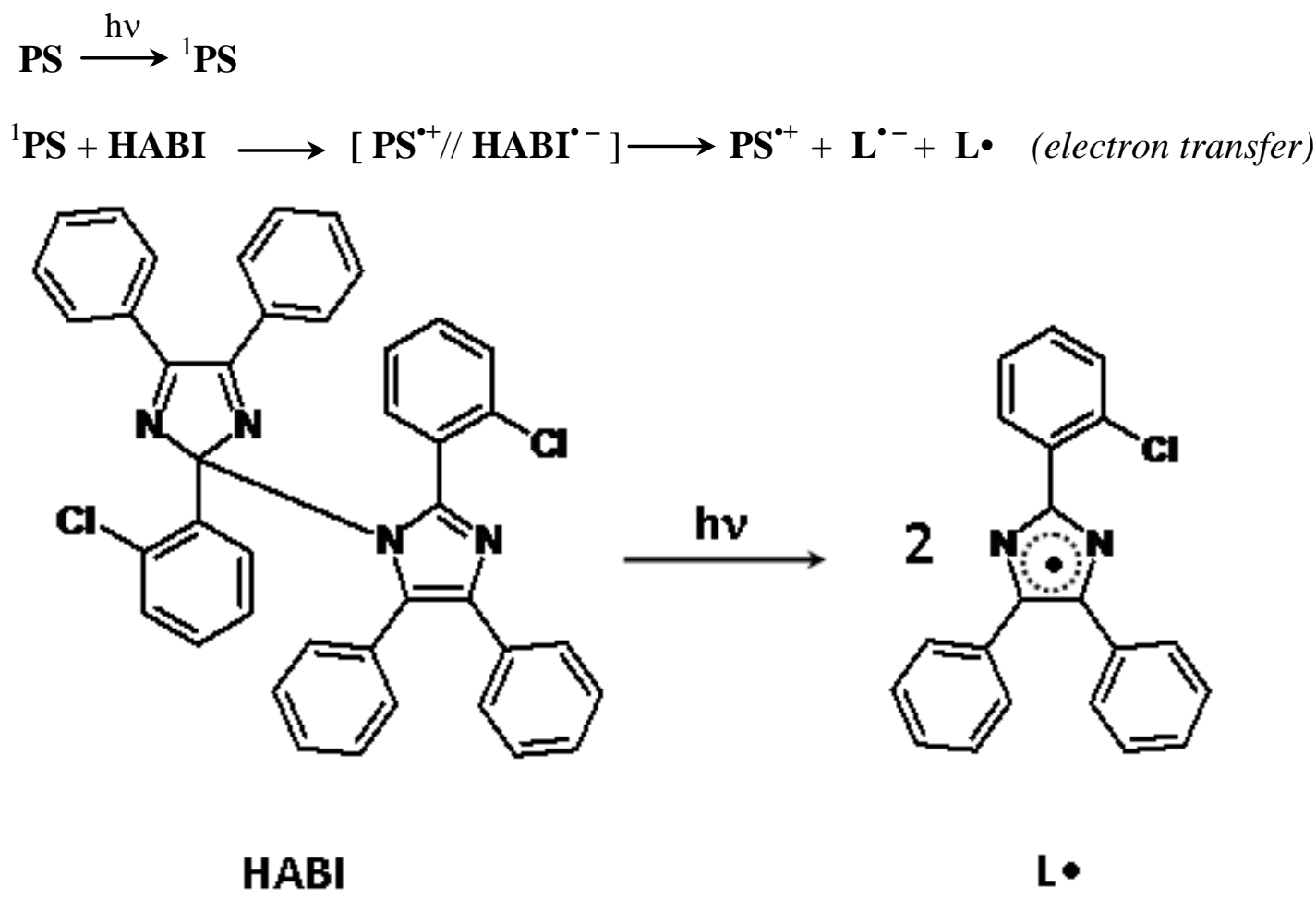

Scheme 4. Homolytic photocleavage process of HABI.

In our case, the assumption of a photoinduced electron transfer at $S_{1}$ state is both supported by the steady-state fluorescence quenching of PS in presence of HABI associated to a decrease of the fluorescence lifetimes in dichloromethane. The Stern-Volmer plots ${ }^{41}$ obtained from the time-resolved measurements lead to linear correlations as depicted in Figure S5. The bimolecular quenching rate constant have values of ca. $4.0 \times 10^{9} \mathrm{M}^{-1} \mathrm{~s}^{-1}$ and ca. $5.8 \times 10^{9} \mathrm{M}^{-1}$ $\mathrm{s}^{-1}$ for $\mathbf{M}$ and $\mathbf{O g}$ respectively. It should be noted that the photoinduced electron transfer (PeT) process can also occur from the lowest triplet state of the sensitizers. However for both chromophores, the PeT from $\mathrm{T}_{1}$ states is presumably a minor route since excited chromophores mainly deactivate through fluorescence pathways. Moreover, the photosensitization of HABI through a triplet-triplet energy transfer mechanism should be excluded due to the relatively high triplet energy of $\mathbf{H A B I}$ whose value, $2.93 \mathrm{eV},{ }^{37 \mathrm{~b}}$ is already similar to $S_{1}$ state energies of both sensitizers. Therefore, both chromophores constitute interesting one-photon sensitizers for free radical polymerization in the visible range. As shown in Figure 5, the maximum polymerization rate of the formulation with $\mathbf{O g}$ is 1.5 -fold 
higher than that measured for $\mathbf{M}$ even though the $\mathbf{O g}$-formulation clearly exhibits a weaker absorbance at the irradiation wavelength. This effect suggests that $\mathbf{O g}$ should lead to a more efficient photoinitiation process. Indeed, the initiation efficiency $\Phi_{i}$ of a photoinitiating system can be connected to the maximum polymerization rate $\mathrm{R}_{p}$ by the following relationship: ${ }^{7 \mathrm{a}}$

$$
\Phi_{i}=K_{1} \frac{R_{p}^{2}}{I_{a b s}}
$$

Where $K_{l}$ is a global parameter which comprises the concentration of the acrylate monomer as well as the rate constants for the propagation and the termination steps of the polymerization reaction. $\mathrm{I}_{a b s}$ corresponds to the amount of light absorbed by the sample. As the maximum polymerization rate $\left(\mathrm{R}_{p}\right)$ is observed at the initial steps of photopolymerization, we assume that $\mathrm{I}_{a b s}$ is reasonably proportional to $\left(1-10^{-\mathrm{A} 0}\right)$ where $\mathrm{A}_{0}$ denotes the initial absorbance of the formulation. Under this approach, we can show that the initiation efficiency of $\mathbf{O g}$ is 3.5 higher as compared to that of $\mathbf{M}$. This higher reactivity can be also demonstrated locally upon two-photon polymerization. Figure 6 shows several polymerized lines generated upon two-photon excitation at $800 \mathrm{~nm}$ using the formulations with $\mathbf{M}$ and $\mathbf{O g}$. Note that the photopolymerization is not observed without the presence of sensitizers which obviously underlines the two-photon activation role of PS. The incident laser power is gradually decreased to estimate the power threshold $\left(\mathrm{P}_{t h}\right)$ necessary to induce the two-photon polymerization. This fundamental parameter should be related the minimal amount of $\alpha-$ aminoalkyl radicals $\left(\mathrm{n}_{t h}\right)$ that effectively lead to the polymerization reaction. Due to the equivalent photoinitiation mechanism for $\mathbf{M}$ and $\mathbf{O g}$, the value for $\mathrm{n}_{t h}$ should remain constant for both formulations and the initiation efficiency $\Phi_{i}$ can be expressed as follows: ${ }^{42}$

$$
\Phi_{i}=K_{2} \frac{n_{t h}}{\delta \cdot N_{P S} \cdot P_{t h}^{2}}
$$

Where $K_{2}$ is a global parameter which implies all the photonic conditions: exposure time, pulse shape parameter, laser temporal pulse width, and repetition rate of the laser. $\mathrm{N}_{P S}$ and $\delta$ are the number density of the photoinitiator and the 2PA cross section. As shown in Figure 6, the values for $\mathrm{P}_{t h}$ are about $5 \mathrm{~mW}$ and $3.6 \mathrm{~mW}$ for $\mathbf{M}$ and $\mathbf{O g}$ respectively which leads to a 2-fold higher initiation efficiency for $\mathbf{O g}$ with respect to $\mathbf{M}$. Therefore, both one- and twophoton polymerization measurements nicely converge to the same conclusion that the photosensitization of the bicomponent system HABI/MDEA exhibits a much higher reactivity when using $\mathbf{O g}$. It is clear that the efficiency for the generation $\alpha$-aminoalkyl 
radicals strongly depends on the amount of $\mathbf{L} \cdot$ used as hydrogen abstractors. However, the back electron transfer $(\mathrm{BeT})$ between the primary generated $\mathbf{P S}^{{ }^{++}} / \mathbf{H A B I}^{--}$radical ion pair can clearly impact the efficiency of all subsequent reactions. As a consequence, the electronic stabilization of $\mathbf{P S}^{\boldsymbol{O}^{+}}$is of prime importance to counterbalance such a radical ion pair recombination. Even though $\mathbf{O g}$ presents a distorted geometry, its amine centered radical cation $\mathbf{O g}^{\circ+}$ should present a more planar conformation around its electron-deficient nitrogen atom $^{44}$ allowing an extended charge delocalization along two adjacent subunits which presumably guarantees a better stabilization as compared to $\mathbf{M}^{\bullet+}$. Therefore, the better stability of $\mathbf{O g}^{\circ+}$ with respect to $\mathbf{M}^{\circ+}$ should indirectly promote a higher quantum yield for the generation of $\mathbf{L} \bullet$. Hence, with the use of $\mathbf{O g}$ as a two-photon sensitizer one clearly promotes a synergy effect with a high photoinitiation reactivity and very large two-photon absorption cross-section $\left(\delta_{\mathrm{MAX}}=5093 \mathrm{GM}\right)$.

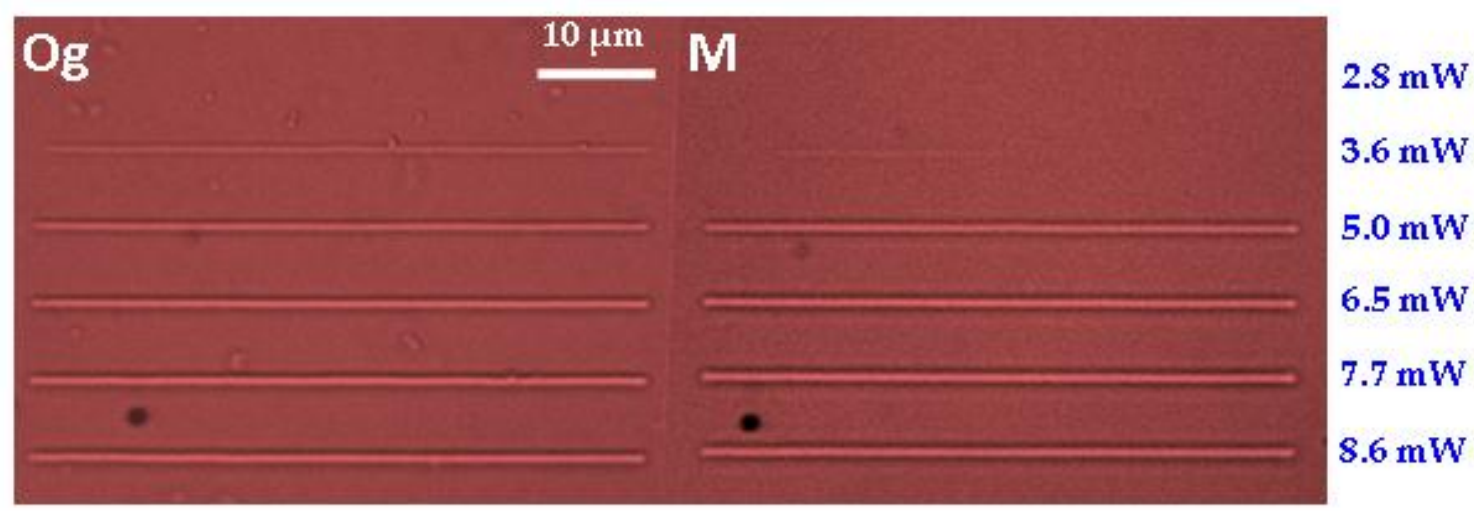

Figure 6. Polymer lines fabricated at various laser exposure powers $\left(\lambda_{\text {exc }}: 800 \mathrm{~nm}\right.$, $\tau_{\text {exp }}=10 \mathrm{~ms}$ ) using diacrylate resins with HABI/MDEA : $1.5 \mathrm{wt} \% / 3 \mathrm{wt} \%$ and containing 2PA sensitizers $\mathbf{O g}(\mathrm{c}=0.09 \mathrm{mM})$ and $\mathbf{M}(\mathrm{c}=2 \mathrm{mM})$.

Finally, to qualitatively demonstrate the potential applications of this multi-component system for 2PA stereolithography, we produced a 2D microstructure by exciting the resin with $\mathbf{O g}$ at $800 \mathrm{~nm}$. The Figure 7 shows the scanning electron micrograph of the 2D microstructure fabricated at an average power of $4 \mathrm{~mW}$. The pattern is impressed into the resin by the relative translation of the laser focus within the material then the final structure is obtained by dissolving away the unexposed formulation. The formation of a two-dimensional microstructure with an arbitrary complexity was thus achieved with good-fidelity replication of the master model displayed in inset of Figure 7. 


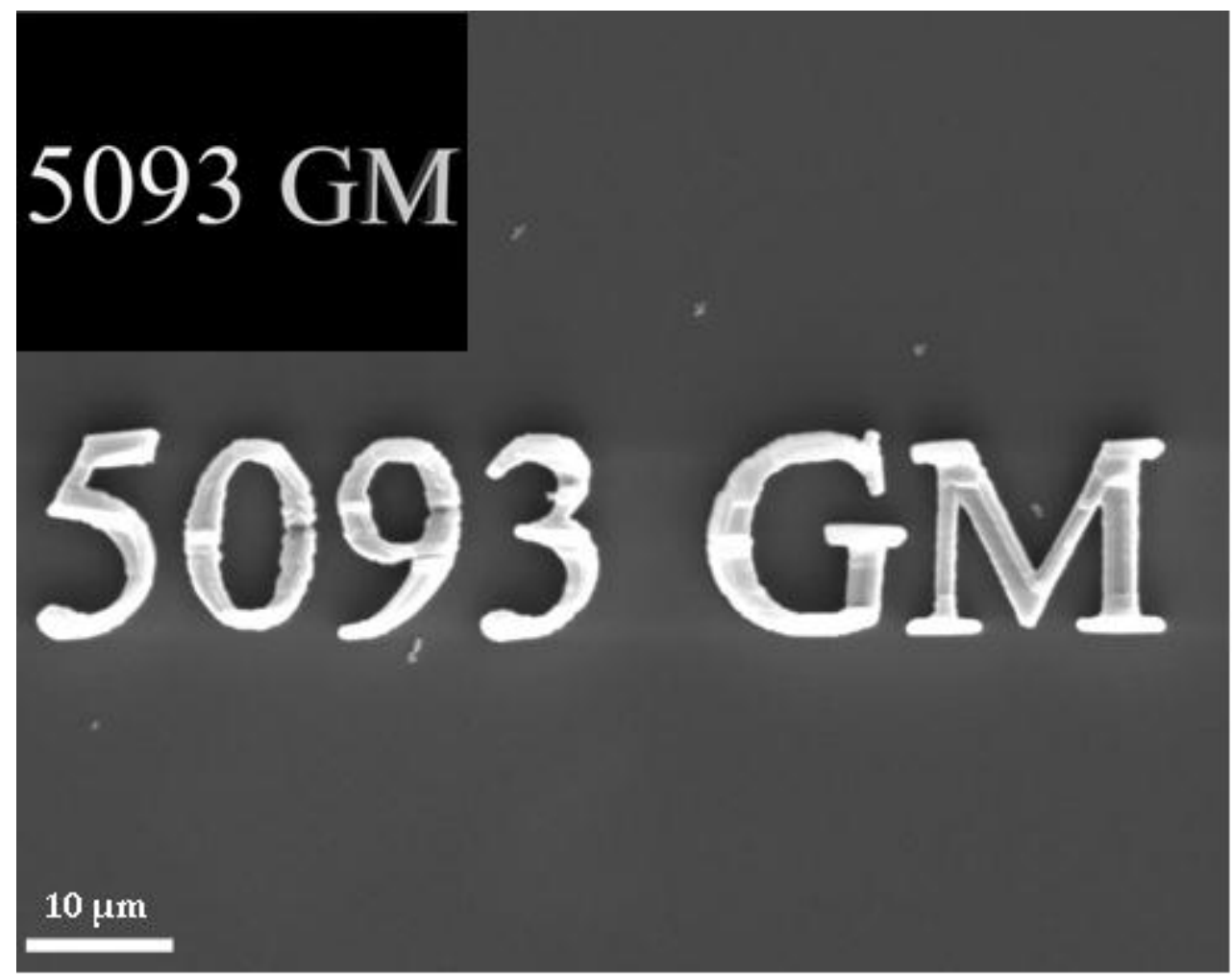

Figure 7. SEM image of a $\mu$-structure fabricated upon excitation at $800 \mathrm{~nm}$ of the diacrylate resin with $\mathbf{O g}$ as $2 \mathrm{PA}$ sensitizer. Inset: 2D model master.

\section{CONCLUSION}

The synthesis of a new conjugated oligomer alternating 4,6-bis(styryl)pyrimidine and Nphenylamine subunits has been reported. The oligomer exhibits very high one- and twophoton absorption ability with corresponding spectra that strikingly resemble those measured for its D- $\pi$-A V-shaped monomer. These spectral effects are ascribed to the highly distorted geometry of the oligomer along its $\pi$-conjugated backbone which reduces the effective conjugation length. Therefore $\mathbf{O}_{\mathbf{g}}$ can be compared to a composite macromolecule which preserves the electronic integrity of each M-unit due to a weak interchromophore coupling. The recursive implementation of several D- $\pi$-A subunits into a three-dimensional architecture leads to a $\delta_{\text {MAX }}$ of about 5093 GM. Taking advantage of the very large linear and non-linear absorption properties of the oligomer, we show that this new system can be used as an efficient sensitizer for free-radical photopolymerization and multiphoton fabrication. The photoinitiation reactivity is two-times higher than its model chromophore which demonstrates that increasing dimensionality not only induces a cooperative enhancement of the nonlinear optical response but can specifically enhance the photopolymerization performance due to the 
further stabilization of intermediate species involved in the photoinitiation mechanism. Such a synergetic strategy can be presumably extended to other $\pi$-conjugated oligomers with comparable symmetries.

\section{EXPERIMENTAL SECTION}

\subsection{Materials and General Characterization Methods.}

The starting materials were acquired from Sigma-Aldrich or Acros and used without purification. In air and moisture-sensitive reactions, all glassware was flame-dried and cooled under nitrogen. All the solvents employed for absorption and emission analysis were Aldrich or Fluka spectroscopic grade. The absorption and fluorescence of all solvents were checked for impurities and have been subtracted from the sample spectra. Ebecryl 605, a difunctional monomer with a high viscosity $\left(\eta \sim 1000 \mathrm{cps}\right.$ at $\left.25^{\circ} \mathrm{C}\right)$, was purchased from Cytec. This viscous monomer used for one- and two-photon polymerization is a formulation based on bisphenol A epoxydiacrylate and tripropyleneglycol diacrylate (75/25 in weight). The 2,2'di(orthochlorophenyl)-4,4',5,5'-tetraphenyl biimidazole (HABI) was purchased from Tokyo Chemical Industry Company.

NMR spectra were recorded in $\mathrm{CDCl}_{3}$, with a Bruker DRX500 spectrometer. $\left({ }^{1} \mathrm{H}\right.$ at 500 $\mathrm{MHz}$ and ${ }^{13} \mathrm{C}$ at $125 \mathrm{MHz}$ ) at the "Service Commun de Recherche de Résonance Magnétique Nucléaire et de Résonance Paramagnétique Electronique de l'Université de Bretagne Occidentale". The chemical shifts $\delta$ are reported in ppm relative to the residual solvent $\left(\mathrm{CHCl}_{3}\right)$. The coupling constants $J$ are given in $\mathrm{Hz}$. In the ${ }^{1} \mathrm{H}$ NMR spectra, the following abbreviations are used to describe peak pattern: s (singlet), d (doublet), dd (doublet of doublet), $\mathrm{t}$ (triplet), $\mathrm{m}$ (multiplet), In the ${ }^{13} \mathrm{C}$ NMR spectra, the nature of the carbons $(\mathrm{C}, \mathrm{CH}$, $\mathrm{CH}_{2}$ or $\mathrm{CH}_{3}$ ) was determined by recording the JMOD experiment. Elemental analyses were performed at the "Centre Régional de Mesures Physiques de l'Ouest" (CRMPO, University or Rennes 1) using a Thermo-Finnigan Flasch EA 1112 CHNS/0 analyser. Analytical thin layer chromatographies (TLC) were performed on silica gel plates (Merk 60F254) and compounds were visualized by irradiation with UV light at 254 and $365 \mathrm{~nm}$. Flash column chromatography was performed using silica gel silica Acros SI 60 (60-200 mesh ASTM). Average molar mass $\left(M_{n}\right)$ and molar mass distribution $\left(M_{w}\right)$ values of the oligomer were 
determined by size-exclusion chromatography (SEC) in THF at $30^{\circ} \mathrm{C}$ (flow rate $1.0 \mathrm{~mL} . \mathrm{min}^{-1}$ ) on a Polymer Laboratories PL50 apparatus equipped with a refractive index detector and a ResiPore $300 \times 7.5 \mathrm{~mm}$ column. The oligomer sample was dissolved in THF $\left(2 \mathrm{mg} \cdot \mathrm{mL}^{-1}\right)$. The elution curve was calibrated with polystyrene standards.

\subsection{Synthesis of chromophores.}

Synthesis of 4,6-Dimethyl-2-octyloxy-pyrimidine (3) (see Scheme 2). To a solution of octan-1-ol $(630 \mu \mathrm{L}, 4.0 \mathrm{mmol})$ in $20 \mathrm{~mL}$ of anhydrous THF under nitrogen at $0^{\circ}$ was added $\mathrm{n}$ $\operatorname{BuLi}(2.5 \mathrm{M}$ in hexanes, $1.6 \mathrm{~mL}$ ) dropwise. The reaction mixture was stirred at room temperature for $30 \mathrm{~min}$. then a solution of 2-chloro-4,6-dimethylpyrimidine (427 $\mathrm{mg}, 3.0$ $\mathrm{mmol}$ ) in $10 \mathrm{~mL}$ of $\mathrm{THF}$ was added dropwise, the reaction mixture was heated to reflux with stirring overnight. The reaction was quenched with water $(20 \mathrm{~mL})$ diluted with $20 \mathrm{~mL}$ of $\mathrm{CH}_{2} \mathrm{Cl}_{2}$ and the organic layer separated. The aqueous layer was extracted with $\mathrm{CH}_{2} \mathrm{Cl}_{2}(2 \times 20$ $\mathrm{mL}$ ). The combined organic extracts were dried over magnesium sulfate and evaporated. The crude product was purified by column chromatography (silica gel, eluent petroleum ether:ethyl acetate (7:3)) to give $475 \mathrm{mg}(67 \%)$ of 3 as a colorless oil. ${ }^{1} \mathrm{H}$ NMR $(500 \mathrm{MHz}$, $\left.\mathrm{CDCl}_{3}\right) \delta 0.85(\mathrm{t}, 3 \mathrm{H}, J=7 \mathrm{~Hz}), 1.31-1.25(\mathrm{~m}, 10 \mathrm{H}), 1.52-1.53(\mathrm{~m}, 2 \mathrm{H}), 1.79-1.73(\mathrm{~m}, 2 \mathrm{H})$, $2.36(\mathrm{~s}, 6 \mathrm{H}), 4.28(\mathrm{t}, J=6.5 \mathrm{~Hz}, 2 \mathrm{H}), 6.61(\mathrm{~s}, 1 \mathrm{H}) ;{ }^{13} \mathrm{C} \mathrm{NMR}$ and JMOD $\left(125 \mathrm{MHz}, \mathrm{CDCl}_{3}\right) \delta$ $169.0(\mathrm{C}), 165.2(\mathrm{C}), 113.6(\mathrm{CH}), 67.3\left(\mathrm{CH}_{2}\right), 31.8\left(\mathrm{CH}_{2}\right), 29.4\left(\mathrm{CH}_{2}\right), 29.2\left(\mathrm{CH}_{2}\right), 29.0\left(\mathrm{CH}_{2}\right)$, $26.0\left(\mathrm{CH}_{2}\right), 22.6\left(\mathrm{CH}_{2}\right), 14.1\left(\mathrm{CH}_{3}\right)$. Anal. Calcd for $\mathrm{C}_{14} \mathrm{H}_{24} \mathrm{~N}_{2} \mathrm{O}: \mathrm{C}, 71.14 ; \mathrm{H}, 10.23 ; \mathrm{N}$, 11.85. Found C, 70.85; H, 10.48; N, 11.59.

Synthesis of oligomer Og. A stirred mixture of $3(118 \mathrm{mg}, 0.5 \mathrm{mmol})$ and 4 (150 mg, 0.5 $\mathrm{mmol})$ in aqueous sodium hydroxide $(5 \mathrm{M}, 10 \mathrm{~mL})$ containing Aliquat 336 (13 mg, 0.03 mmol) was heated under reflux for $3 \mathrm{~h}$. The mixture was allowed to cool, and the precipitate was filtered off, washed with water, and purified by precipitation from $\mathrm{CHCl}_{3} / \mathrm{n}$-heptane giving $242 \mathrm{mg}(94 \%)$ as a red solid. ${ }^{1} \mathrm{H} \mathrm{NMR}\left(500 \mathrm{MHz}, \mathrm{CDCl}_{3}\right) \delta 0.86(\mathrm{t}, 3 \mathrm{H}, J=7 \mathrm{~Hz})$, 1.31-1.25 (m, 8H), 1.52-1.53 (m, 2H), 1.79-1.73 (m, 2H), $4.47(\mathrm{t}, J=6 \mathrm{~Hz}, 2 \mathrm{H}), 6.87(\mathrm{~s}, 1 \mathrm{H})$, $6.90(\mathrm{~d}, 2 \mathrm{H}, J=15.5 \mathrm{~Hz}), 7.17-7.09(\mathrm{~m}, 7 \mathrm{H}), 7.32-7.30(\mathrm{~m}, 2 \mathrm{H}), 7.49$ (d, 4H, $J=8 \mathrm{~Hz}), 7.86$ $(\mathrm{d}, 2 \mathrm{H}, J=15.5 \mathrm{~Hz}) ;{ }^{13} \mathrm{C}$ NMR and JMOD $\left(125 \mathrm{MHz}, \mathrm{CDCl}_{3}\right) \delta 165.5(\mathrm{C}), 165.0(\mathrm{C}), 148.2$ (C), $146.7(\mathrm{C}), 136.0(\mathrm{CH}), 130.4(\mathrm{C}), 129.6(\mathrm{CH}), 128.8(\mathrm{CH}), 125.7(\mathrm{CH}), 125.1(\mathrm{CH})$, $124.3(\mathrm{CH}), 123.6(\mathrm{CH}), 67.5\left(\mathrm{CH}_{2}\right), 31.9\left(\mathrm{CH}_{2}\right), 29.5\left(\mathrm{CH}_{2}\right), 29.3\left(\mathrm{CH}_{2}\right), 29.1\left(\mathrm{CH}_{2}\right), 26.1$ 
$\left(\mathrm{CH}_{2}\right), 22.7\left(\mathrm{CH}_{2}\right), 14.1\left(\mathrm{CH}_{3}\right)$. Anal. Calcd for $\left(\mathrm{C}_{35} \mathrm{H}_{37} \mathrm{~N}_{3} \mathrm{O}\right)_{\mathrm{n}}: \mathrm{C}, 81.52 ; \mathrm{H}, 7.23 ; \mathrm{N}, 8.15$. Found C, 81.60; H, 7.54; N, 7.76.

\subsection{Instrumentation and Methods.}

The absorption measurements were carried out with a Perkin Elmer Lambda 2 double beam UV-vis spectrophotometer. Steady-state fluorescence spectra were collected from a FluoroMax-4 spectrofluorometer. Emission spectra are spectrally corrected, and fluorescence quantum yields include the correction due to solvent refractive index and were determined relative to quinine bisulfate in 0.05 molar sulfuric acid $(\Phi=0.52) .{ }^{44}$

For the steady-state anisotropy measurements, two Glan-Thompson polarizers are placed in the excitation and emission beams. The anisotropy $r$ is determined as follows:

$$
r=\frac{I_{V V}-g I_{V H}}{I_{V V}+2 \cdot g \cdot I_{V H}} \quad \text { with } g=\frac{I_{H V}}{I_{H H}}
$$

Where I is the fluorescence intensity, the subscripts denote the orientation (horizontal $\mathrm{H}$ or vertical $\mathrm{V}$ ) of the excitation and emission polarizers, respectively and $\mathrm{g}$ is an instrumental correction factor. The proper calibration of the set-up was checked using a recent standard method with rhodamine 101 in glycerol. ${ }^{45}$ The anisotropy spectra were collected in viscous medium (i.e. triacetin, $\eta_{20^{\circ} \mathrm{C}}=25 \mathrm{mPa} . \mathrm{s}$ ) in order to prevent the depolarization of the fluorescence emission due to the rotational diffusion of the molecules. ${ }^{41}$

The fluorescence lifetimes were measured using a Nano LED emitting at $372 \mathrm{~nm}$ as an excitation source with a nano led controller module, Fluorohub from IBH, operating at $1 \mathrm{MHz}$. The detection was based on an R928P type photomultiplier from Hamamatsu with high sensitivity photon-counting mode. The decays were fitted with the iterative reconvolution method on the basis of the Marquardt/Levenberg algorithm. ${ }^{46}$ Such a reconvolution technique allows an overall-time resolution down to $0.15 \mathrm{~ns}$. The quality of the exponential fits was checked using the reduced $\chi^{2}(\leq 1.2)$.

The two-photon absorption (2PA) measurements were performed with femtosecond modelocked laser pulse using a Ti: Sapphire laser (Coherent, Chameleon Ultra II: pulse duration: 
140 fs; repetition rate: $80 \mathrm{MHz}$; wavelength range: 680-1040 nm). A relative two-photon excited fluorescence (2PEF) method ${ }^{31}$ was employed to measure the two-photon absorption cross-sections, $\delta$. The measurements of 2PA cross-sections were performed relative to reference molecules $(r)$ such as fluorescein ${ }^{31,47}$ in water at $\mathrm{pH}=11$. The value of $\delta$ for a sample $(S)$ is given by:

$$
\delta_{S}=\frac{S_{S} \Phi_{r} \eta_{r} c_{r}}{S_{r} \Phi_{S} \eta_{S} c_{S}} \cdot \delta_{r}
$$

Where $S$ is the detected two-photon excited fluorescence integral area, $c$ the concentration of the chromophores, and $\Phi$ is the fluorescence quantum yield of the chromophores. $\eta$ is the collection efficiency of the experimental set-up and accounts for the wavelength dependence of the detectors and optics as well as the difference in refractive indices between the solvents in which the reference and sample compounds are dissolved. The measurements were conducted in a regime where the fluorescence signal showed a quadratic dependence on the intensity of the excitation beam, as expected for two-photon induced emission. For the calibration of the two-photon absorption spectra, the two-photon excited fluorescence signal of each compound was recorded at the same excitation wavelength as that used for standards (i.e. $\lambda_{\text {exc }}=782 \mathrm{~nm}$ for fluorescein). The laser intensity was in the range of $0.2-2 \times 10^{9} \mathrm{~W} / \mathrm{cm}^{2}$. The experimental error on the reported cross section is $15 \%$.

The photopolymerization was monitored in situ by Fourier transform real-time infrared spectroscopy (FT-RTIR) with a Thermo-Nicolet 6700 instrument IR-spectrometer. The laminated formulations are sandwiched between two polypropylene films (10 $\mu \mathrm{m}$ thick) separated by a round teflon spacer of $25 \mu \mathrm{m}$ thickness. The sample is then positioned between two $\mathrm{BaF}_{2}$ pellets and irradiated at $440 \mathrm{~nm}$ using $100 \mathrm{~W}$ Mercury-Xenon Lamp (Hamamatsu, L2422-02) equipped with a band pass filter (FB440-10 from Thorlabs). The conversion rates are obtained from the disappearance of the progressive vinyl $\mathrm{C}=\mathrm{C}$ stretching vibration band at $1630 \mathrm{~cm}^{-1}$.

The 3D lithographic microfabrication was carried out using a Zeiss Axio Observer D1 inverted microscope. The two-photon excitation was performed at $800 \mathrm{~nm}$ using respectively a mode-locked Ti: Sapphire oscillator (Coherent, Chameleon Ultra II: pulse duration: $~ 140 \mathrm{fs;}$ repetition rate: $80 \mathrm{MHz}$ ). The incident beam was focused through a 0.65 NA objective $(40 \mathrm{X})$ which leads to a radial spot size $600 \mathrm{~nm}$ at $\lambda_{\mathrm{exc}}=800 \mathrm{~nm}\left(1 / \mathrm{e}^{2}\right.$ Gaussian). A drop of the resin 
is deposited on a cover slip which is mounted on a 3D piezoelectric stage allowing the translation relative to the laser focal point. The intensity of the incoming laser is controlled with the use of an acoustooptic modulator. The displacement of the sample and all photonic parameters (i.e. excitation power and irradiation times) are computer-controlled. This entire lithography set-up was purchased from Teemphotonics Company.

The theoretical absorption spectra have been computed based on Density Functional Theory (DFT) and Time-Dependent DFT (TDDFT). The overall computation strategy was defined as follows: After initial AM1 optimization calculations (vacuum), subsequent optimization of geometrical structures of the derivatives were carried out using the PBE0/6$31 \mathrm{G}(\mathrm{d})$ level of calculation. (Note that PBE0 is a good functional for the description of photofunctional molecules ${ }^{48}$ ). Finally, the TDDFT vertical transitions have been computed using the same level of calculations. All calculations have been performed using GAUSSIAN 09 package. $^{49}$

X-ray powder diffraction (XRD) measurements were performed on a D2 Phaser Bruker AXS equipped with a Linxeye detector using a Ni-filtered $\mathrm{CuK} \alpha$ radiation $(\lambda=0.15406 \mathrm{~nm})$. XRD was operated at an accelerating voltage of $30 \mathrm{kV}$ and a current of $10 \mathrm{~mA}$ with the $2 \theta$ angle ranging from $5^{\circ}$ to $40^{\circ}$ at the scanning speed of $0.8^{\circ} / \mathrm{min}\left(0.02^{\circ}\right.$ intervals $)$. Refractive indices were measured at room temperature using a Prism Coupler (Model: 2010/M, Metricon) at three different wavelengths $(\lambda=632.8,1320$ and $1550 \mathrm{~nm})$.

\section{ACKNOWLEDGEMENTS}

We thank the meso-centre 'EQUIPEX@MESO' of Strasbourg University for access to HPC resources. We also gratefully thank Dr Sophie Guillaume and Cedric Jaffredo, from Institut des Sciences Chimiques de Rennes for SEC analysis. Dr Christine Baudequin from Université de Rouen as well as Adrien Morrelec-Le Men and Benoît Quinton, students at IUT Lannion, are acknowledged for preliminary synthesis and optical measurements. Bresmat network is also acknowledged for funding.

\section{REFERENCES}


1. (a) B. H. Cumpston, S. P.; Ananthavel, S. Barlow, D. L. Dyer, J. E. Ehrlich, L. L. Erskine, A. A. Heika, S. M. Kuebler, I.-Y. S. Lee, D. McCord-Maughon, J. Qin, H. Röckel, M. Rumi, X.-L. Wu, S. R. Marder and J. W. Perry, Nature, 1999, 398, 51-54. (b) S. Kawata, H. B. Sun, T. Tanaka and K. Takada, Nature, 2001, 412, 697-698. (c) H. B. Sun and S. Kawata, Two-Photon Photopolymerization and 3D Lithographic Microfabrication; Springer-Verlag ed. Berlin, 2004; Vol. 170.

2. (a) H. A. Collins, M. Khurana, E. H. Moriyama, A. Mariampillai, E. Dahlstedt, M. Balaz, M. K. Kuimova, M. Drobizhev, V. X. D. Yang, D. Phillips, A. Rebane, B. C. Wilson and H. L. Anderson Nature Photonics, 2008, 2, 420-424. (b) J. R. Starkey, A. K. Rebane, M. A. Drobizhev, F. Meng, A. Gong, A. Elliott, K. McInnerney and C. W. Spangler Clin. Cancer Res. 2008, 14, 6564-6573. (c) G. Garcia, F. Hammerer, F. Poyer, S. Achelle, M.-P. Teulade-Fichou and P. Maillard Bioorg. Med. Chem. 2013, 21, 153-165.

3. (a) S. Yao and K. D. Belfield, Eur. J. Org. Chem. 2012, 3199-3217. (b) S. Sumalekshmy and C. J. Fahrni, Chem. Mater. 2011, 23, 483-500. (c) M. Drobizhev, N. S. Makarov, S. E. Tillo, T. E. Hughes and A. Rebane Nature Meth. 2011, 8, 393-399.

4. (a) C. N. LaFratta, J. T. Fourkas, T. Baldacchini and R. A. Farrer Angew. Chem. Int. Ed. Engl. 2007, 46, 6238-6258. (b) K. D. Belfield, X. Ren, E. W. V. Stryland, D. J. Hagan, V. Dubikovsky and E. J. Miesak J. Am. Chem. Soc. 2000, 122, 1217-1218. (c) K. D. Belfield and K. J. Schafer, Chem. Mater. 2002, 14, 3656-3662. (d) M. Jin, J.-P. Malval, D. L. Versace, F. Morlet-Savary, H. Chaumeil, A. Defoin, X. Allonas and J. P. Fouassier, Chem. Comm. 2008, 48, 6540-6542. (e) J.-P. Malval, M. Jin, F. Morlet-Savary, H. Chaumeil, A. Defoin, O. Soppera, T. Scheul, M. Bouriauand P. L. Baldeck Chem. Mater. 2011, 23, 3411-3420. (f) J.-P. Malval, F. Morlet-Savary, H. Chaumeil, L. Balan, D.-L. Versace, M. Jin and A. Defoin $J$. Phys. Chem. C 2009, 113, 20812-20821. (g) R. Xia, J.-P. Malval, M. Jin, A. Spangenberg, D. Wan, H. Pu, T. Vergote, F. Morlet-Savary, H. Chaumeil, P. Baldeck, O. Poizat and O. Soppera Chem. Mater. 2012, 24, 237-244.

5. K. J. Schafer, J. M. Hales, M.; Balu, K. D. Belfield, E. W. V. Stryland and D. J. Hagan J. Photochem. Photobio. A: Chem. 2004, 162, 497-502.

6. (a) J. Kabatc and J. Paczkowski Macromolecules 2005, 38, 9985-9992. (b) K.-S. Lee, D.-Y. Yang, S. H. Park and R. H. Kim Polym. Adv. Technol. 2006, 17, 72-82. (c) K.-S. Lee, R. H. Kim, D.-Y. Yang and S. H. Park Prog. Polym. Sci. 2008, 33, 631-681. (d) H. Yuan, Y. Zhao and F. Wu Chem. Mater. 2012, 24, 1371-1377. (e) K. D. Belfield K. J. Schafer, Y. Liu, J. Liu, X. Ren and E. W. V. Stryland J. Phys. Org. Chem. 2000, 13, 837-849. (f) C. N. LaFratta, T. Baldacchini, R. A. Farrer, J. T. Fourkas, M. C. Teich, B. E. A. Saleh and M. J. Naughton J. Phys. Chem. B 2004, 108, 11256-11258.

7. (a) J. P. Fouassier, Photoinitiation, Photopolymerization, and Photocuring; Hanser: Munich, 1995. (b) M. Shirai and M. Tsunooka Prog. Polym. Sci. 1996, 21, 1-45.

8. (a) G. S. He, L.-S. Tan, Q Zheng and P. N. Prasad, Chem. Rev. 2008, 108, 1245-1330. (b) M. Pawlicki, H. A. Collins, R. G. Denning and H. L. Anderson Angew. Chem. Int. Ed. 2009, 48, 3244-3266.

9. (a) D. Beljonne, W. Wenseleers, E. Zojer, Z. Shuai, H. Vogel, S. J. K. Pond, J. W. Perry, S. R. Marder and J. L. Brédas Adv. Funct. Mater. 2002, 12, 631-641. (b) F. Terenziani, C. Katan, E. Badaeva, S. Tretiak and M. Blanchard-Desce, Adv. Mater. 2008, 20, 4641-4678. (c) M. Rumi, J. E. Ehrlich, A. Heikal, J. W. Perry, S. Barlow, Z. Y. Hu, D. McCord-Maughon, T. C. Parker, H. Rockel, S. Thayumanavan, S. R. Marder, D. Beljonne and J. L. Brédas J. Am. Chem. Soc. 2000, 122, 9500-9510. (e) C. Katan, F. Terenziani, O. Mongin, M. H. V. Werts, L.Porrès, T. Pons, J. Mertz, S. Tretiak and M. Blanchard-Desce J. Phys. Chem. A 2005, 109, 3024-3037. (f) C. Katan, S. Tretiak, M. H. V. Werts, A. J. Bain, R. J. Marsh, N. Leonczek, N. Nicolaou, E. Badaeva, O. Mongin and M. Blanchard-Desce J. Phys. Chem. B 2007, 111, 9468-9483. (g) M. Johnsen, M. J. Paterson, J. Arnbjerg, O. Christiansen, C. B. Nielsen, M. Jorgensen and P. R. Ogilby Phys. Chem. Chem. Phys. 2008, 10, 1177-1191.

10. P. Savel, H. Akdas-Kilig, J.-P. Malval, A. Spangenberg, T. Roisnel and J.-L. Fillaut J. Mater. Chem. C 2014, 2, 295-305. 
11. (a) S.-J. Chung, M. Rumi, V. Alain, S. Barlow, J. W. Perry and S. R. Marder J. Am. Chem. Soc. 2005, 127, 10844-10845. (b) F. Stellacci, C. A. Bauer, T. Meyer-Friedrichsen, W. Wenseleers, S. R. Marder and J. W. Perry J. Am. Chem. Soc. 2003, 125, 328-329.

12. (a) M. Drobizhev, A. Karotki, Y. Dzenis, A. Rebane, Z. Suo and C. W. Spangler J. Phys. Chem. B 2003, 107, 7540-7543. (b) M. Drobizhev, A. Rebane, Z. Suo and C. W. Spangler J. Lumin. 2005, 111, 291-305. (c) O. Varnavski, X. Yan, O. Mongin, M. Blanchard-Desce and T. Goodson J. Phys. Chem. C 2006, 111, 149-162.

13. G. P. Bartholomew, M. Rumi, S. J. K. Pond, J. W. Perry, S. Tretiak and G. C. Bazan J. Am. Chem. Soc. 2004, 126, 11529-11542.

14. (a) R. E. Martin and F. Diederich Angew. Chem. Int. Ed. Engl. 1999, 38, 1350-1377. (b) X. Li, X. Zhang, W. Li, Y. Wang, T. Liu, B. Zhang and W. Yang J. Mater.Chem. 2011, 21, 39163924. (c) M. G. Vivas, S. L. Nogueira, H. S. Silva, N. M. Barbosa Neto, A. Marletta, F. SereinSpirau, S. Lois, T. Jarrosson, L. De Boni, R. A. Silva and C. R. Mendonca J. Phys. Chem. B 2011, 115, 12687-12693. (d) J. Pina and J. S. Seixas de Melo Phys. Chem. Chem. Phys. 2009, 11, 8706-8713. (e) H. Meier Angew. Chem. Int. Ed. Engl. 2005, 44, 2482-2506.

15. (a) P. Najechalski, Y. Morel, O. Stéphan and P. L. Baldeck Chem. Phys. Lett. 2001, 343, 44-48.

(b) Y. Morel, O. Stéphan, C. Andraud and P. L. Baldeck Synth. Met. 2001, 124, 237-239.

16. M. Williams-Harry, A. Bhaskar, G. Ramakrishna, T. Goodson, M. Imamura, A. Mawatari, K. Nakao, H. Enozawa, T. Nishinaga and M. Iyoda, J. Am. Chem. Soc. 2008, 130, 3252-3253.

17. M.-C. Yoon, S. B. Noh, A. Tsuda, Y. Nakamura, A. Osuka, D. Kim, J. Am. Chem. Soc. 2007, 129, 10080-10081.

18. (a) J. M. Fréchet, J. Polym. Sci. Part A: Polym. Chem. 2003, 41, 3713-3725. (b) J. C. GarcíaMartínez, E. Díez-Barra and J. Rodríguez-López Curr. Org. Synth. 2008, 5, 267-290.

19. S. Achelle, I. Nouira, B. Pfaffinger, Y. Ramondenc, N. Plé and J. Rodríguez-López J. Org. Chem. 2009, 74, 3711-3717.

20. J.-J. Vanden Eynde, L. Pascal, Y. Van Haverbeke and P. Dubois Synth. Commun. 2001, 31, 3167-3173.

21. (a) C. Hadad, S. Achelle, J. C. García-Martínez, J. Rodríguez-López J. Org. Chem. 2011, 76, 3837-3845. (b) A. I. Aranda, S. Achelle, F. Hammerer, F. Mahuteau-Betzer and M.-P. TeuladeFichou Dyes Pigm. 2012, 95, 400-407. (c) S. Achelle, A. Barsella, C. Baudequin, B. Caro and F. Robin-le Guen J. Org. Chem. 2012, 77, 4087-4096. (d) S. Achelle and F.; Robin-le Guen Tetrahedron Lett. 2013, 54, 4491-4496.

22. A. S. Davidov, Theory of molecular excitons; Plenum Press: New York, 1971.

23. S. Achelle, J.-P. Malval, S. Aloïse, A. Barsella, A. Spangenberg, L. Mager, H. Akdas-Kilig, J.L. Fillaut, B. Caro and Robin-le Guen Chem. Phys. Chem. 2013, 14, 2725-2736.

24. (a) R. S. Becker, J. Seixas de Melo, A. L. Maçanita and F. Elisei J. Phys. Chem. 1996, 100, 18683-18695. (b) A. Facchetti, M.-H. Yoon, C. L. Stern, G. R. Hutchison, M. A. Ratner and T. J. Marks, J. Am. Chem. Soc. 2004, 126, 13480-13501.

25. (a) N. Aratani, A. Osuka, Y. H. Kim, D. H. Jeong and D. Kim, Angew. Chem. Int. Ed. Engl. 2000, 39, 1458-1462. (b) N. W. Song, H. S. Cho, M. C. Yoon, N. Aratani, A. Osuka and D. Kim, Bull. Korean Chem. Soc. 2002, 23, 271-276. (c) D. H. Yoon, S. B. Lee, K. H. Yoo, J. Kim, J. K. Lim, N. Aratani, A. Tsuda, A. Osuka and D. Kim J. Am. Chem. Soc. 2003, 125, 11062-11064. (d) H. S. Cho, D. H. Jeong, S. Cho, D. Kim, Y. Matsuzaki, K. Tanaka, A. Tsuda and A. Osuka J. Am. Chem. Soc. 2002, 124, 14642-14654.

26. (a) J.-S. Yang, S.-Y. Chiou and K.-L. Liau J. Am. Chem. Soc. 2002, 124, 2518-2527. (b) M. Jin, H. Hong, J. Xie, J.-P. Malval, A. Spangenberg, O. Soppera, D. Wan, H. Pu, D.-L. Versace, T. Leclerc, P. L. Baldeck, O. Poizat and S. Knopf Polym. Chem. 2014, DOI: 10.1039/C4PY00424H

27. J. R. Platt, J. Chem. Phys. 1949, 17, 484-487.

28. R. P. Subrayan, J. W. Kampf and P. G. Rasmussen J. Org. Chem. 1994, 59, 4341-4345.

29. (a) J. Rotzler, D. Vonlanthen, A. Barsella, A. Boeglin, A. Fort and M. Mayor Eur. J. Org. Chem. 2010, 1096-1110. (b) J.-P. Malval, V. Diemer, F. Morlet-Savary, P. Jacques, H. Chaumeil, A. Defoin, C. Carre and O. Poizat J. Phys. Chem. A 2010, 114, 2401-2411.

30. M. Kasha, H. R. Rawls and M. El-Bayoumi Pure Appl. Chem. 1965, 11, 371-392.

31. C. Xu and W. W. Webb J. Opt. Soc. Am. B 1996, 13, 481-491. 
32. (a) M. G. Kuzyk J. Chem. Phys. 2003, 119, 8327-8334. (b) J. P. Moreno and M. G. Kuzyk J. Chem. Phys. 2005, 123, 194101.

33. K. Nakao, M. Nishimura, T. Tamachi, Y. Kuwatani, H. Miyasaka, T. Nishinaga and M. Iyoda $J$. Am. Chem. Soc. 2006, 128, 16740-16747.

34. (a) R. Dessauer In Advances in Photochemistry; John Wiley \& Sons, Inc.: 2005, p 129-261. (b) B. M. Monroe and G. C. Weed Chem. Rev. 1993, 93, 435-448.

35. (a) H. Miyasaka, Y. Satoh, Y. Ishibashi, S. Ito, Y. Nagasawa, S. Taniguchi, H. Chosrowjan, N. Mataga, D. Kato, A. Kikuchi and J. Abe J. Am. Chem. Soc. 2009, 131, 7256-7263. (b) A. D. Liu, A. D. Trifunac and V. V. Krongauz J. Phys. Chem. 1992, 96, 207-211.

36. (a) M. Kawano, T. Sano, J. Abe and Y. Ohashi J. Am. Chem. Soc. 1999, 121, 8106-8107. (b) J. V. Caspar, I. V. Khudyakov, N. J. Turro and G. C. Weed Macromolecules 1995, 28, 636-641.

37. (a) D. F. Eaton, A. Gafney Horgan and J. P. Horgan J. Photochem. Photobiol. A 1991, 58, 373-391. (b) S. Suzuki, T. Urano, K. Ito, T. Murayama, I. Hotta, S. Takahara and T. Yamaoka J. Photopolym. Sci. Tech. 2004, 17, 125-130. (c) S. Suzuki, T. Urano, N. Miyagawa, S. Takahara and T. Yamaoka J. Photopolym. Sci. Tech. 2001, 14, 259-262.

38. H. Fischer and L. Radom Angew. Chem. Int. Ed. Engl. 2001, 40, 1340-1371.

39. (a) D. D. M. Wayner, J. J. Dannenberg and D. Griller Chem. Phys. Lett. 1986, 131, 189-191. (b) D. D. M. Wayner and D. Griller J. Am. Chem. Soc. 1985, 107, 7764-7765. (c) J.-P. Malval, M. Jin, L. Balan, R. Schneider, D.-L. Versace, H. Chaumeil, A. Defoin and O. Soppera J. Phys. Chem. C 2010, 114, 10396-10402.

40. (a) Y. Lin, A. Liu, A. D. Trifunac and V. V. Krongauz Chem. Phys. Lett. 1992, 198, 200-206.

(b) X. Li, Y. Zhao, J. Wu, M. Shi and F. Wu J. Photochem. Photobiol. A 2007, 190, 22-28. (c) Y. Zhao, X. Li, F. Wu and X. Fang J. Photochem. Photobiol. A 2006, 177, 12-16. (d) S. Berdzinski, N. Strehmel, H. Lindauer, V. Strehmel and B. Strehmel Photochem. Photobiol. Sci. 2014, DOI : 10.1039/C3PP50379H

41. J. R. Lakowicz Principles of Fluorescence Spectroscopy; Third ed.; Springer Science: New York, 2006.

42. (a) C. Martineau, R. Anémian, C. Andraud, I. Wang, M. Bouriau and P. L. Baldeck Chem.Phys.Lett. 2002, 362, 291-295. (b) C. Martineau, G. Lemercier, C. Andraud, I. Wang, M. Bouriau and P. L. Baldeck Synth. Met. 2003, 138, 353-356. (c) S. A. Pruzinsky and V. Braun Adv. Funct. Mater. 2005, 15, 1995-2004.

43. (a) A. Yella, R. Humphry-Baker, B. F. E. Curchod, N. Ashari Astani, J. Teuscher, L. E. Polander, S. Mathew, J.-E. Moser, I. Tavernelli, U. Rothlisberger, M. Grätzel, M. K. Nazeeruddin and J. Frey Chem. Mater. 2013, 25, 2733-2739. (b) J.-P. Malval, J.-P. Morand, R. Lapouyade, W. Rettig, G. Jonusauskas, J. Oberle, C. Trieflinger and J. Daub Photochem. Photobiol. Sci. 2004, 3, 939-948. (c) J.-P. Malval, R. Lapouyade, J.-M. Leger and C Jarry Photochem. Photobiol Sci. 2003, 2, 259-266.

44. R. Meech and D. Phillips J. Photochem. 1983, 23, 193-217.

45. T. J. V. Prazeres, A. Fedorov, S. P. Barbosa, J. M. G. Martinho and M. N. Berberan-Santos J. Phys. Chem. A 2008, 112, 5034-5039.

46. D. V. Connor and D. Phillips Time correlated single photon counting, Academic Press: London, 1984.

47. M. A. Albota, C. Xu and W. W. Webb Appl. Opt. 1998, 37, 7352-7356.

48. S. Aloïse, Z. Pawlowska, C. Ruckebusch, M. Sliwa, J. Dubois, O. Poizat, G. Buntinx, A. Perrier, F. Maurel, P. Jacques, J.-P. Malval, L. Poisson, G. Piani and J. Abe Phys. Chem. Chem. Phys. 2012, 14, 1945-1956.

49. M. J. Frisch, In Gaussian 09, Revision B.01, Gaussian, Inc., Wallingford CT Wallingford CT, 2009. 